\title{
Conformational analysis of the potential anticancer agent ethyl trihydroxycinnamate-A combined raman spectroscopy and ab initio study
}

\author{
J.B. Sousa a ${ }^{\text {a }}$ R. Calheiros ${ }^{\text {a }}$, V. Rio ${ }^{\text {b }}$, F. Borges ${ }^{\text {a,b }}$, M.P.M. Marques ${ }^{\text {a,c, }, *}$ \\ ${ }^{a}$ Unidade I\&D 'Química-Física Molecular', Faculdade de Ciências e Tecnologia, Universidade de Coimbra, 3000 Coimbra, Portugal

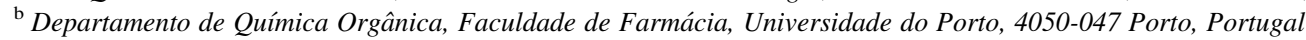 \\ ${ }^{\mathrm{c}}$ Departamento de Bioquímica, Faculdade de Ciências e Tecnologia, Universidade de Coimbra, Ap. 3126, 3001-401 Coimbra, Portugal
}

Received 13 July 2005; revised 31 August 2005; accepted 6 September 2005

Available online 17 October 2005

\begin{abstract}
A conformational analysis of ethyl 3-(3,4,5-trihydroxyphenyl)-2-propenoate (ethyl 3,4,5-trihydroxycinnamate, ETHPPE), a polyphenolic cinnamic ester which displays antiproliferative activity towards human adenocarcinoma cells, was carried out by Raman spectroscopy coupled to ab initio MO calculations. Apart from the optimised geometrical parameters for the most stable conformations of this compound (both for the trans and cis isomers), the corresponding harmonic vibrational frequencies were obtained. Eighteen distinct geometries were found, 12 for the lowest energy trans isomer and six for the cis species. The conformational preferences of this system were verified to be mainly ruled by the stabilising effect of $\pi$-electron delocalisation, a planar geometry being favoured. The orientation of the ester moiety showed to be the most determinant factor for the overall stability of the molecule. In the light of these results, a complete assignment of the corresponding Raman pattern was performed.
\end{abstract}

(C) 2005 Elsevier B.V. All rights reserved.

Keywords: Ethyl trihydroxycinnamate; Raman spectroscopy; Ab initio calculations; Conformational analysis

\section{Introduction}

Phenolic acid derivatives are a group of compounds, present in the human diet in significative amounts, which display an enormous variety of biological functions [1-13], such as antioxidant activity, anti-inflammatory action, and carcinogenesis modulation (e.g. due to their prooxidant capacity that may induce oxidative damage of DNA, proteins or cell lipids). Epidemiological studies linking the prevalence of certain diseases to dietary patterns often show an inverse correlation between a specific diet (particularly rich in leafy green vegetables) and certain pathologic states, namely neoplastic disorders $[14,15]$. The cytotoxic activity of phenolic systems, closely related to their antioxidant

\footnotetext{
* Corresponding author. Address: Departamento de Bioquímica, Faculdade de Ciências e Tecnologia, Universidade de Coimbra, Ap. 3126, 3001-401 Coimbra, Portugal. Tel.: +351 239854462; fax: +351 239826541.

E-mail address: pmc@ci.uc.pt (M.P.M. Marques).
}

properties [3,16-18], is ruled by their structural characteristics (which determine their lipophilicity and degree of incorporation into the cells). The nature of the biological target, the environmental conditions and the phenol dosage and bioavailability are also factors influencing the therapeutic ability of this kind of compounds.

The evaluation of the antioxidant/antiproliferative activity of phenolic derivatives, either of natural or synthetic origin, aiming at the development of new and more effective anticancer drugs, is nowadays an important area of research in the field of Medicinal Sciences. Indeed, numerous studies have been carried out in order to find new leader compounds (e.g. structurally based on benzoic and cinnamic acids $[19,20]$ ), suitable for obtaining either cancer chemopreventive or chemotherapeutic agents. Even though there is a wealth of data on the relevance of phenolic compounds as growthinhibition agents, the correlation between anticancer activity and chemical structure is far from clear. In fact, no thorough structure-activity relationships (SAR's) may be drawn from the literature, as no systematic study was performed to this date. One of the problems is the scattering of the reported data, which results from different methods of assessment, varying 
substrate systems and miscellaneous concentrations of putative antiproliferative compounds.

The hydroxycinnamic acid derivative trans-3-(3,4,5-trihydroxyphenyl)-2-propenoic acid (THPPE) and its esters transethyl(3,4,5-trihydroxyphenyl)-2-propenoate (ETHPPE) and diethyl 2-(3,4,5-trihydroxyphenylmethylene)malonate (E2THPPE) (Fig. 1) have lately been synthesised and evaluated for their antioxidant and anticancer activities [21]. The monoester ETHPPE was found to display significant growthinhibition and cytotoxic effects towards a human cervix adenocarcinoma cell line (HeLa), along with a low toxicity against non-neoplastic cells (human skin fibroblasts), in accordance with reported data for the analogous caffeic and gallic esters [22,23]. Marked structure-activity relationships (SAR's) were found to rule the antioxidant and anticancer activities of these hydroxycinamic acid derivatives. Actually, their biological functions were determined to be intrinsically dependent on the number and relative orientation of the ring hydroxyl substituents, as well as on the presence of alkyl ester side chains and their chemical nature and spatial orientation (e.g. linear vs branched, saturated vs unsaturated). Therefore, the knowledge of these conformational preferences is of the utmost importance for the understanding and/or prediction of the antioxidant and anticancer properties of this kind of systems.

In the present work, a complete conformational analysis was performed for trans-ethyl(3,4,5-trihydroxyphenyl)-2-propenoate (ETHPPE), by Raman spectroscopy coupled to ab initio MO calculations (at the DFT level). A comparison with the analogous diester diethyl 2-(3,4,5-trihydroxyphenylmethylene) malonate (E2THPPE) was carried out. This study was intended as a continuation of the one previously reported for the corresponding 3-(3,4,5-trihydroxy-phenyl)-2-propenoic acid (THPPE) [24].

\section{Experimental}

\subsection{Synthesis}

Trans-3-(3,4,5-Trihydroxyphenyl)-2-propenoic acid (3,4,5trihydroxycinnamic acid, THPPE) and trans-ethyl 3-(3,4, 5-trihydroxyphenyl)-2-propenoate (trans-ethyl 3,4,5-trihydroxycinnamate, ETHPPE) were synthesised as described elsewhere [24]. The compounds were identified by both NMR and EI-MS. The data obtained is in accordance with that described in [24].

Diethyl 2-(3,4,5-trihydroxyphenylmethylene)malonate (E2THPPE) was synthesised according to the process described by Hubner et al. [25] with slight modifications. Yield: 70\%; IR: 3388, 3282, 1720, 1672, 1600, 1535, 1465, 1375,1326, 1272, 1234, 1149, 1078, 1032, 939, 837, 766, 731, 665, 631. ${ }^{1} \mathrm{H}$ NMR $\delta: 1.23\left(6 \mathrm{H}, t, \mathrm{COOCH}_{2} \mathrm{CH}_{3}\right), 4.19(2 \mathrm{H}, q$, $\left.\mathrm{COOCH}_{2} \mathrm{CH}_{3}\right), 4.30\left(2 \mathrm{H}, q, \mathrm{COOCH}_{2} \mathrm{CH}_{3}\right), 6.48(2 \mathrm{H}, s, \mathrm{H}(2)$, $\mathrm{H}(6)), 7.37(1 \mathrm{H}, s, \mathrm{H}(\beta)), 9.20(3 \mathrm{H}, s, \mathrm{OH}) ;{ }^{13} \mathrm{C}$ NMR $\delta: 13.8$ $\left(\mathrm{OCH}_{2} \mathrm{CH}_{3}\right), 14.1 \quad\left(\mathrm{OCH}_{2} \mathrm{CH}_{3}\right), 61.1 \quad\left(\mathrm{OCH}_{2} \mathrm{CH}_{3}\right), 61.3$ $\left(\mathrm{OCH}_{2} \mathrm{CH}_{3}\right), 109.4(\mathrm{CH}(2,6)), 121.7(\mathrm{C}(1)), 122.3(\mathrm{C}(\alpha)$, $137.2(C-\mathrm{OH}), 142.0(C \mathrm{H}(\beta)), 146.0(C-\mathrm{OH}), 164.0(\mathrm{C}=\mathrm{O})$, $166.6(\mathrm{C}=\mathrm{O})$; EI-MS $m / z(\%): 296\left(\mathrm{M}^{+} \cdot, 100\right), 251$ (36), 222 (38), 205 (49), 178 (38), 150 (58), mp $182-184{ }^{\circ} \mathrm{C}\left(156{ }^{\circ} \mathrm{C}\right.$ subl.).

Infrared spectra were recorded on a ATI Mattson Genesis Series FTIR spectrophotometer, using potassium bromide disks. Only the most significant absorption bands are reported $\left(\nu_{\max }, \mathrm{cm}^{-1}\right) .{ }^{1} \mathrm{H}$ and ${ }^{13} \mathrm{C} \mathrm{NMR}$ data were acquired at room temperature, on a Brüker AMX 300 spectrometer operating at 300.13 and $75.47 \mathrm{MHz}$, respectively. DMSO- $d_{6}$ was used as a solvent; chemical shifts are expressed in $\delta$ (ppm) values relative to tetramethylsilane (TMS) as an internal reference;
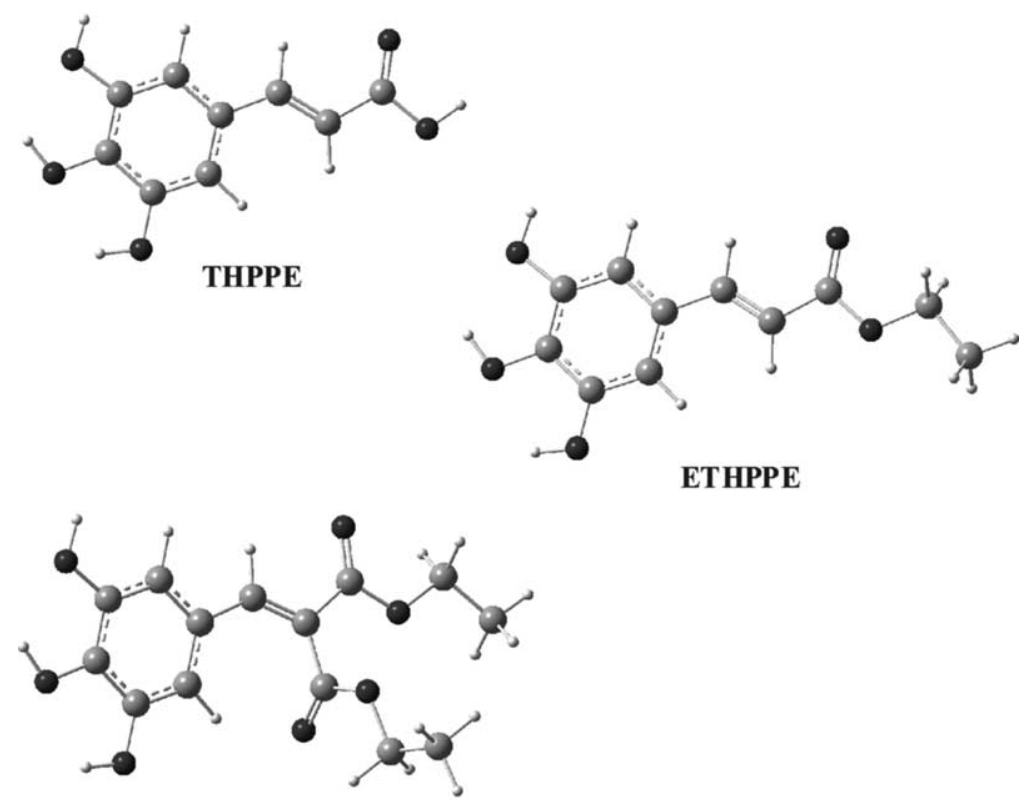

E2THPPE

Fig. 1. Representation of the most stable conformers calculated for trans-3-(3,4,5-trihydroxyphenyl)-2-propenoic acid (THPPE) and its esters trans-ethyl(3,4,5trihydroxyphenyl)-2-propenoate (ETHPPE) and diethyl 2-(3,4,5-trihydroxy-phenylmethylene)malonate (E2THPPE). (B3LYP/6-31G** level of calculation). 
coupling constants $(J)$ are given in Hz. Assignments were also made from distortionless enhancement by polarization transfer (DEPT) (underlined values). Electron impact mass spectra (EIMS) were carried out on a VG AutoSpec instrument; the data are reported as $\mathrm{m} / \mathrm{z}$ (\% of relative intensity of the most important fragments). Melting points were obtained on a Köfler microscope (Reichert Thermovar) and are uncorrected.

\subsection{Ab initio $M O$ calculations}

The ab initio calculations-full geometry optimisation and calculation of the harmonic vibrational frequencies-were performed using the GAUSSIAN $98 \mathrm{~W}$ program [26], within the Density Functional Theory (DFT) approach, in order to properly account for the electron correlation effects (particularly important in this kind of conjugated systems). The widely employed hybrid method denoted by B3LYP, which includes a mixture of HF and DFT exchange terms and the gradientcorrected correlation functional of Lee, Yang and Parr [27,28], as proposed and parametrised by Becke [29,30], was used, along with the double-zeta split valence basis set $6-31 \mathrm{G}^{* *}$ [31]. Wavenumbers above $400 \mathrm{~cm}^{-1}$ were scaled by a factor of 0.9614 [32] before comparing them with the experimental data. The basis set superposition error (BSSE) correction for the dimerisation energies was estimated by counterpoise (CP) calculations [33], using the MASSAGE option of GAUSSIAN 98W.

Molecular geometries were fully optimised by the Berny algorithm, using redundant internal coordinates [34]: The bond lengths to within ca. $0.1 \mathrm{pm}$ and the bond angles to within ca. $0.1^{\circ}$. The final root-mean-square (rms) gradients were always less than
$3 \times 10^{-4}$ hartree bohr ${ }^{-1}$ or hartree radian ${ }^{-1}$. No geometrical constraints were imposed on the molecules under study.

\subsection{Raman spectroscopy}

The Raman spectra of the ETHPPE solutions (in Dimethylsulfoxide, DMSO) were obtained at room temperature, in a triple monochromator Jobin-Yvon T64000 Raman system $(0.640 \mathrm{~m}, f / 7.5)$ with holographic gratings of 1800 grooves $\mathrm{mm}^{-1}$. The detection system was a nonintensified CCD (Charge Coupled Device) and the entrance slit was set to $200 \mu \mathrm{m}$. The $514.5 \mathrm{~nm}$ line of an $\mathrm{Ar}^{+}$laser (Coherent, model Innova 300) was used as excitation radiation, providing ca. $50 \mathrm{~mW}$ at the sample position. Samples were sealed in Kimax glass capillary tubes of $0.8 \mathrm{~mm}$ inner diameter. Under the above mentioned conditions, the error in wavenumbers was estimated to be within $1 \mathrm{~cm}^{-1}$.

Fourier transform Raman spectra were recorded for the solid samples (in order to avoid fluorescence), in a RFS 100/S Bruker spectrometer with a $180^{\circ}$ geometry, equipped with an InGaAs detector. Near-infrared excitation was provided by the $1064 \mathrm{~nm}$ line of a Nd:YAG laser (Coherent, model Compass$1064 / 500 \mathrm{~N})$. A laser power of $200 \mathrm{~mW}$ at the sample position was used in all cases, and resolution was set to $2 \mathrm{~cm}^{-1}$.

\subsection{Reagents}

Reagents. 3,4,5-trihydroxyaldehyde, trans-caffeic acid, dimethylsulfoxide, monoethylmalonate, diethylmalonate and malonic acid were purchased from Sigma-Aldrich Química S.A. (Sintra, Portugal). Dimethylsulfoxide- $d_{6}(99.8 \%)$ was obtained from E. Merck, Darmstadt, Germany. All other

(a)
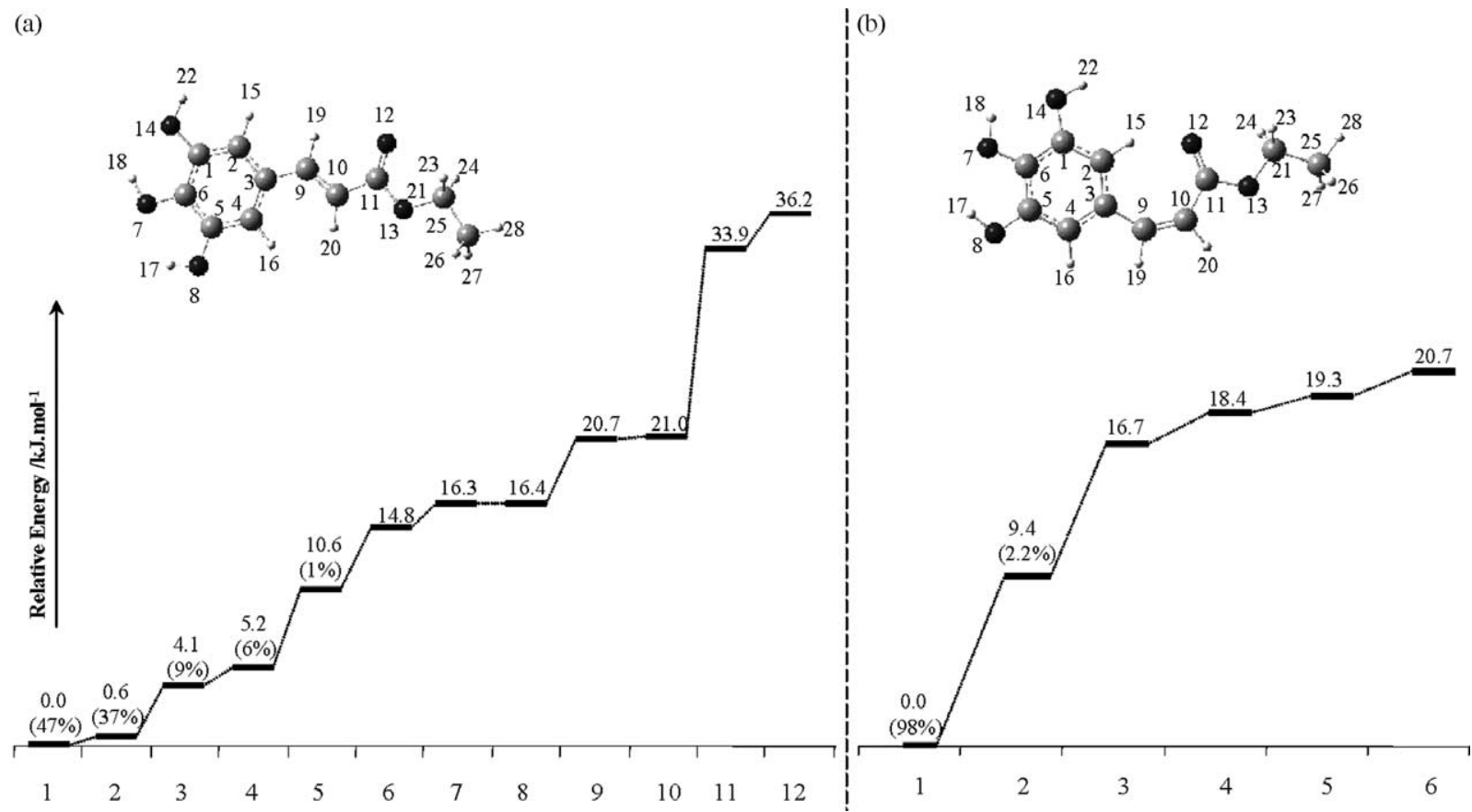

Fig. 2. Schematic representation of the calculated (B3LYP/6-31G**) conformational energies (and populations, at $\left.25^{\circ} \mathrm{C}\right)$ for ethyl 3-(3,4,5-trihydroxyphenyl)-2propenoate (ETHPPE): (a) trans isomer; (b) cis isomer. (The atom numbering is included). 
reagents and solvents were pro analysis grade, purchased from Merck (Lisbon, Portugal).

\section{Results and discussion}

\subsection{Ab initio MO calculations}

A complete conformational analysis was carried out for ethyl 3-(3,4,5-trihydroxy-phenyl)-2-propenoate, through ab initio MO calculations: the geometries, relative energies and populations at room temperature were obtained for the distinct possible conformers, for both the cis and trans ETHPPE isomers (dihedral $\left(\mathrm{C}_{11} \mathrm{C}_{10} \mathrm{C}_{9} \mathrm{C}_{3}\right)$ equal to $0^{\circ}$ or $180^{\circ}$, respectively, Fig. 2). Harmonic vibrational frequencies were calculated for each structure, in order to confirm the convergence to minima in the potential energy surface. The effect of several structural parameters on the overall stability of the molecule was investigated, namely: (i) orientation of the whole $\left(\mathrm{C}_{9}=\mathrm{C}_{10}\right.$-ethyl ester $)$ substituent relative to the aromatic ring-internal rotation around the $\mathrm{C}_{9}-\mathrm{C}_{3}$ bond (dihedral $\left(\mathrm{C}_{10} \mathrm{C}_{9} \mathrm{C}_{3} \mathrm{C}_{2}\right)$ equal to $0^{\circ}$ or $180^{\circ}$ ); (ii) orientation of the ester moiety relative to the aromatic ring-rotation about the $\mathrm{C}_{11}-\mathrm{C}_{10}$ bond (dihedral $\left(\mathrm{O}_{13} \mathrm{C}_{11} \mathrm{C}_{10} \mathrm{C}_{9}\right)$ equal to $0^{\circ}$ or $180^{\circ}$ ); (iii) orientation of the ethyl group relative to the carbonylrotation around $\mathrm{O}_{13}-\mathrm{C}_{11}$ (dihedral $\left(\mathrm{C}_{21} \mathrm{O}_{13} \mathrm{C}_{11} \mathrm{C}_{10}\right)$ equal to $180^{\circ}$ or $0^{\circ}$, defining a S-cis or a S-trans conformation, (a)

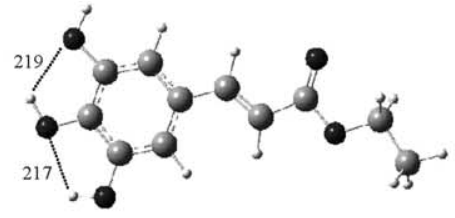

tETHPPE 1

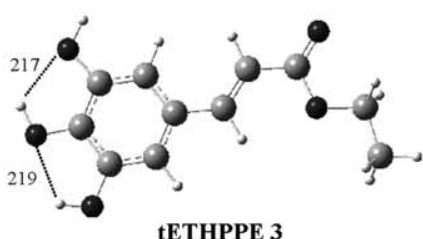

tETHPPE 3

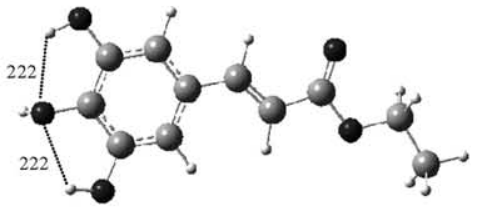

tETHPPE 5

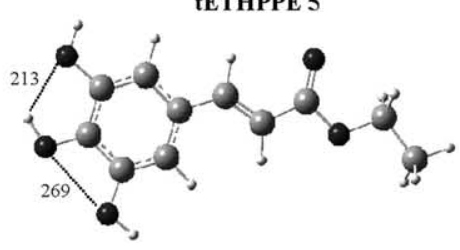

tETHPPE 7
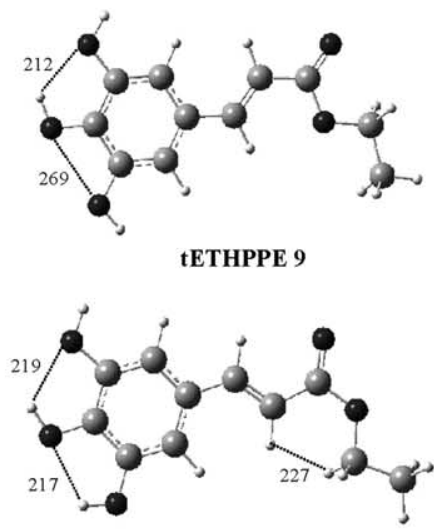

tETHPPE 1

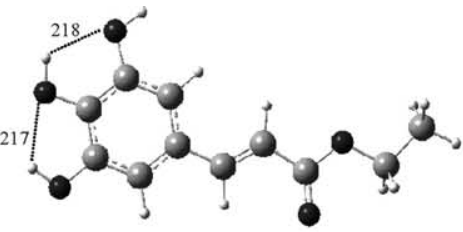

tETHPPE 2

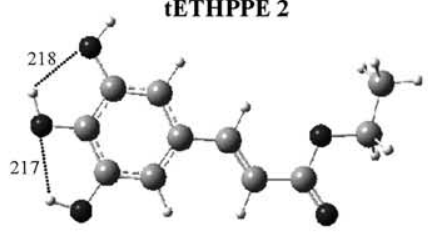

tETHPPE 4

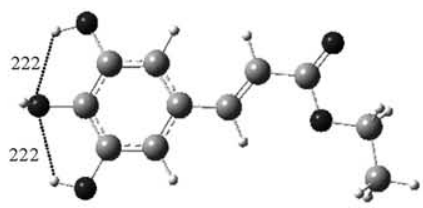

tETHPPE 6

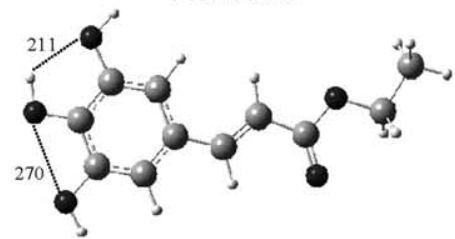

tETHPPE 8
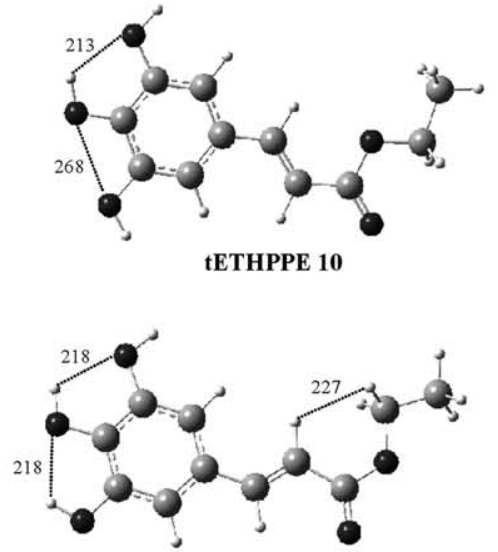

tETHPPE 12

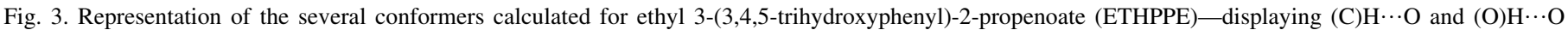
intramolecular interactions. (a) trans isomer; (b) cis isomer. (B3LYP/6-31G** level of calculation. Distances are represented in pm). 

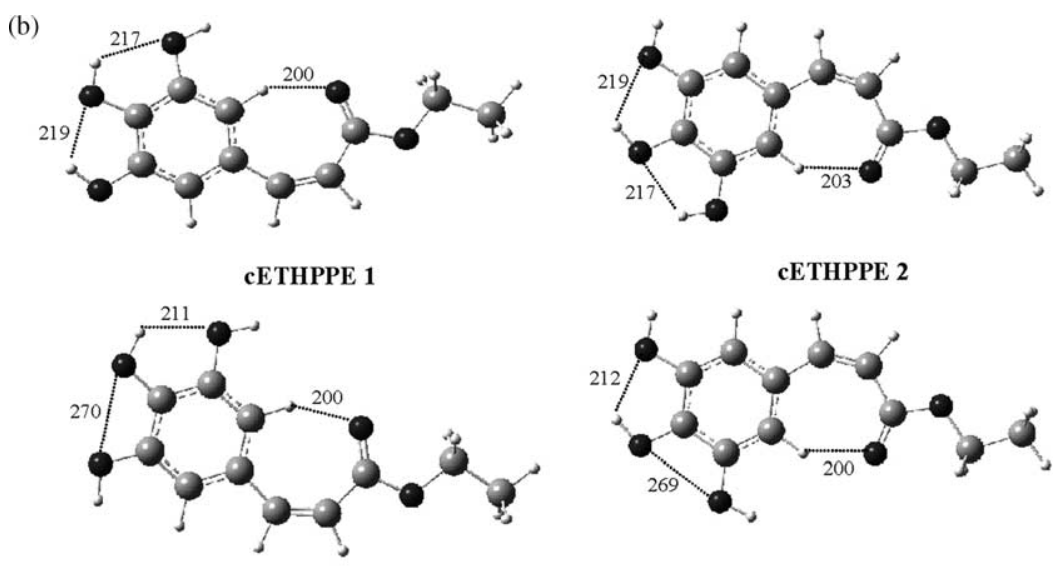

cETHPPE 3
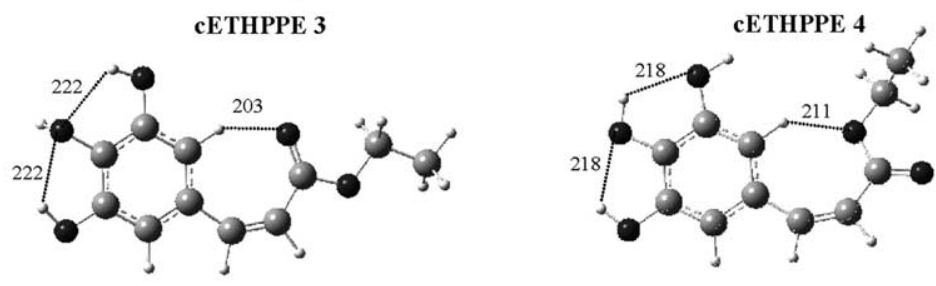

CETHPPE 5

cETHPPE 6

Fig. 3 (continued)

respectively); (iv) conformation of the ethyl group-rotation around the $\mathrm{O}_{13}-\mathrm{C}_{21}$ bond; (v) orientation of the phenolic groups relative to the plane of the ring-either in-plane $\left(\left(\mathrm{H}_{22} \mathrm{O}_{14} \mathrm{C}_{1} \mathrm{C}_{2}\right),\left(\mathrm{H}_{18} \mathrm{O}_{7} \mathrm{C}_{6} \mathrm{C}_{1}\right)\right.$ and $\left(\mathrm{H}_{17} \mathrm{O}_{8} \mathrm{C}_{5} \mathrm{C}_{6}\right)$ equal to $0^{\circ}$ or $180^{\circ}$ ) or out-of-plane. Eighteen different conformers were found for ETHPPE, 12 for the trans isomer (tETHPPE) and six for the cis species (cETHPPE) (Figs. 2 and 3). A planar geometry showed to be favoured due to the stabilising effect of the $\pi$-electron delocalisation between the aromatic ring and the $\mathrm{C}_{9}=\mathrm{C}_{10}$ and $\mathrm{C}_{11}=\mathrm{O}_{12}$ double bonds, which is maximum when they are coplanar. The only non-planar calculated conformers-tETHPPE 5, tETHPPE 6, cETHPPE 5 and cETHPPE 6 -are highly destabilised, tETHPPE 5 being the only one populated at room temperature (1\%) (Fig. 2). tETHPPE 5, tETHPPE 6 and cETHPPE 5 display a central hydroxyl group which is almost perpendicular to the plane of the ring$\left(\mathrm{H}_{18} \mathrm{O}_{7} \mathrm{C}_{6} \mathrm{C}_{5}\right)$ equal to $91.9^{\circ}, 91.3^{\circ}$ and $94.0^{\circ}$, respectivelywhile in cETHPPE 6 the $\left(\mathrm{C}_{9}=\mathrm{C}_{10}\right.$ - ethyl $)$ moiety lies ca. $14.6^{\circ}$ out-of-plane $\left(\left(\mathrm{C}_{10} \mathrm{C}_{9} \mathrm{C}_{3} \mathrm{C}_{2}\right)\right.$ dihedral $)$ and the ethyl ester is tilted by $83.6^{\circ}\left(\left(\mathrm{C}_{25} \mathrm{C}_{21} \mathrm{O}_{13} \mathrm{C}_{11}\right)\right.$ dihedral $)$.

Similarly to what was verified for THPPE [24], the cis isomers of ETHPPE $\left(\left(\mathrm{C}_{11} \mathrm{C}_{10} \mathrm{C}_{9} \mathrm{C}_{3}\right)\right.$ dihedral equal to $\left.0^{\circ}\right)-$ cETHPPE 1-6 (Figs. 2(b) and 3(b)) - display a quite lower stability than the trans species $\left(\left(\mathrm{C}_{11} \mathrm{C}_{10} \mathrm{C}_{9} \mathrm{C}_{3}\right)=180^{\circ}\right.$, Figs. 2 (a) and $3(\mathrm{a})$ ), despite the possible occurrence of intramolecular medium-strength $\mathrm{H}_{15} / \mathrm{H}_{16} \cdots \mathrm{O}_{12}$ interactions in cis-ETHPPE, yielding a seven-membered intramolecular ring $\left(\mathrm{d}\left(\mathrm{H}_{15} / \mathrm{H}_{16} \ldots\right.\right.$ $\mathrm{O}_{12}$ ) between 200 and $211 \mathrm{pm}$ ). Actually, $\pi$-delocalisation is surely more effective in the geometries displaying a linear (zigzag) $\mathrm{C}_{9}=\mathrm{C}_{10}$ - ethyl ester chain (trans geometries). Moreover, the additional stabilisation (by at least $9.4 \mathrm{~kJ} \mathrm{~mol}^{-1}$ ) of cETHPPE 1 as compared to cETHPPE 2 is explained by the higher electronic delocalisation in the former, due to the identical orientation of the ring $\mathrm{OH}$ 's and the $\mathrm{C}=\mathrm{O}$ group. For these cis structures a $\mathrm{C}_{9}-\mathrm{C}_{3}$ rotation proved to be highly unfavourable: cETHPPE $1\left(\left(\mathrm{C}_{10} \mathrm{C}_{9} \mathrm{C}_{3} \mathrm{C}_{2}\right)=0^{\circ}, \Delta E=0\right)$ vs cETHPPE $2\left(\left(\mathrm{C}_{10} \mathrm{C}_{9} \mathrm{C}_{3} \mathrm{C}_{2}\right)=180^{\circ}, \Delta E=9.4 \mathrm{~kJ} \mathrm{~mol}^{-1}\right)$, and cETHPPE $3\left(\left(\mathrm{C}_{10} \mathrm{C}_{9} \mathrm{C}_{3} \mathrm{C}_{2}\right)=0^{\circ}, \Delta E=16.7 \mathrm{~kJ} \mathrm{~mol}^{-1}\right)$ vs cETHPPE $4\left(\left(\mathrm{C}_{10} \mathrm{C}_{9} \mathrm{C}_{3} \mathrm{C}_{2}\right)=180^{\circ}, \Delta E=18.4 \mathrm{~kJ} \mathrm{~mol}^{-1}\right)$. Furthermore, an internal rotation around $\mathrm{C}_{11}-\mathrm{C}_{10}-$ e.g. conversion between conformers cETHPPE 3 and cETHPPE 6 (Fig. 3 (b)) - showed to cause a significant degree of steric hindrance involving the ethyl ester moiety, which resulted in an additional rotation of this group relative to the plane of the aromatic ring $\left(\left(\mathrm{C}_{25} \mathrm{C}_{21} \mathrm{O}_{13} \mathrm{C}_{11}\right)=83.6^{\circ}\right.$ in cETHPPE 6).

Regarding the position of both the $\left(\mathrm{C}_{9}=\mathrm{C}_{10}\right.$ - ester) moiety and the ethyl ester group relative to the benzene ringassociated to the rotations around $\mathrm{C}_{9}-\mathrm{C}_{3}$ and $\mathrm{C}_{11}-\mathrm{C}_{10}$, respectively - those conformations displaying the same orientation of the carbonyl group and the ring $\mathrm{OH}$ substituents (syn conformations) showed to be energetically favoured, on account of a more effective $\pi$-electron delocalisation: tETHPPE 1 $\left(\left(\mathrm{C}_{10} \mathrm{C}_{9} \mathrm{C}_{3} \mathrm{C}_{2}\right)=180^{\circ}, \Delta E=0\right)$ vs tETHPPE $2\left(\left(\mathrm{C}_{10} \mathrm{C}_{9} \mathrm{C}_{3} \mathrm{C}_{2}\right)=\right.$ $\left.0^{\circ}, \Delta E=0.6 \mathrm{~kJ} \mathrm{~mol}^{-1}\right)$, and tETHPPE $3\left(\left(\mathrm{C}_{10} \mathrm{C}_{9} \mathrm{C}_{3} \mathrm{C}_{2}\right)=0^{\circ}\right.$, $\left.\Delta E=4.1 \mathrm{~kJ} \mathrm{~mol}^{-1}\right)$ vs tETHPPE $4\left(\left(\mathrm{C}_{10} \mathrm{C}_{9} \mathrm{C}_{3} \mathrm{C}_{2}\right)=180^{\circ}, \Delta E=\right.$ $\left.5.2 \mathrm{~kJ} \mathrm{~mol}^{-1}\right)$. For $\left(\mathrm{C}_{10} \mathrm{C}_{9} \mathrm{C}_{3} \mathrm{C}_{2}\right)=0^{\circ}$, in turn, the geometries displaying a close-to-linear arrangement of the ester substituent were found to be stabilised: tETHPPE $2\left(\left(\mathrm{O}_{13} \mathrm{C}_{11} \mathrm{C}_{10} \mathrm{C}_{92}\right)=\right.$ $\left.180^{\circ}, \Delta E=0.6 \mathrm{~kJ} \mathrm{~mol}^{-1}\right)$ vs tETHPPE $3\left(\left(\mathrm{O}_{13} \mathrm{C}_{11} \mathrm{C}_{10} \mathrm{C}_{9}\right)=0^{\circ}\right.$, $\left.\Delta E=4.1 \mathrm{~kJ} \mathrm{~mol}^{-1}\right)$ (Figs. 2(a) and 3(a)). Similarly, for $\left(\mathrm{C}_{10} \mathrm{C}_{9^{-}}\right.$ $\left.\mathrm{C}_{3} \mathrm{C}_{2}\right)=180^{\circ}$, conformer tETHPPE $1\left(\left(\mathrm{O}_{13} \mathrm{C}_{11} \mathrm{C}_{10} \mathrm{C}_{9}\right)=180^{\circ}\right.$, $\Delta E=0)$ is favoured relative to tETHPPE $4\left(\left(\mathrm{O}_{13} \mathrm{C}_{11} \mathrm{C}_{10} \mathrm{C}_{9}\right)=0^{\circ}\right.$, $\left.\Delta E=5.2 \mathrm{~kJ} \mathrm{~mol}^{-1}\right)$. This is in agreement with the results previously obtained for THPPE [24]. However, for those 
Table 1

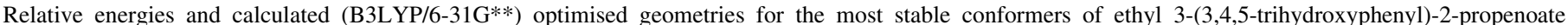
(ETHPPE)

\begin{tabular}{|c|c|c|c|c|}
\hline \multirow[t]{2}{*}{$\Delta E\left(\mathrm{kJmol}^{-1}\right) / \mu(\mathrm{D})^{\mathrm{a}}$} & tETHPPE 1 & cETHPPE 1 & $\mathrm{tTHPPE}^{\mathrm{b}}$ & $\mathrm{CA}^{\mathrm{c}}$ \\
\hline & $0 ; 2.6^{\mathrm{d}}$ & $0 ; 1.9$ & $0 ; 3.5$ & $0 ; 3.5$ \\
\hline \multicolumn{5}{|l|}{ Bond lengths (pm) } \\
\hline $\mathrm{C}_{1}-\mathrm{C}_{6}^{\mathrm{e}}$ & 139.6 & 140.0 & 139.6 & 139.2 \\
\hline $\mathrm{C}_{2}-\mathrm{C}_{1}$ & 139.0 & 138.7 & 139.0 & 139.3 \\
\hline $\mathrm{C}_{3}-\mathrm{C}_{2}$ & 140.8 & 141.0 & 140.9 & 140.5 \\
\hline $\mathrm{C}_{3}-\mathrm{C}_{9}$ & 146.0 & 146.0 & 145.9 & 145.6 \\
\hline $\mathrm{C}_{4}-\mathrm{C}_{3}$ & 140.6 & 141.1 & 140.7 & 141.3 \\
\hline $\mathrm{C}_{5}-\mathrm{C}_{4}$ & 139.0 & 139.1 & 139.0 & 138.3 \\
\hline $\mathrm{C}_{6}-\mathrm{C}_{5}$ & 139.5 & 139.9 & 140.2 & 141.2 \\
\hline $\mathrm{C}_{9}-\mathrm{C}_{10}$ & 134.6 & 135.7 & 134.7 & 134.8 \\
\hline $\mathrm{C}_{10}-\mathrm{C}_{11}$ & 147.5 & 147.1 & 147.2 & 147.0 \\
\hline $\mathrm{C}_{21}-\mathrm{C}_{25}$ & 151.7 & 151.7 & - & - \\
\hline $\mathrm{C}_{1}-\mathrm{O}_{14}$ & 137.5 & 137.6 & 137.5 & - \\
\hline $\mathrm{C}_{5}-\mathrm{O}_{8}$ & 136.8 & 136.3 & 136.1 & 137.5 \\
\hline $\mathrm{C}_{6}-\mathrm{O}_{7}$ & 136.8 & 136.7 & 136.8 & 135.6 \\
\hline $\mathrm{C}_{11}-\mathrm{O}_{12}$ & 121.8 & 122.2 & 121.8 & 121.8 \\
\hline $\mathrm{C}_{11}-\mathrm{O}_{13}$ & 135.7 & 135.9 & 136.1 & 136.2 \\
\hline $\mathrm{C}_{21}-\mathrm{O}_{13}$ & 144.0 & 144.2 & - & - \\
\hline $\mathrm{O}_{14}-\mathrm{H}_{22}$ & 96.5 & 96.6 & 96.5 & - \\
\hline $\mathrm{O}_{7}-\mathrm{H}_{18}$ & 96.9 & 96.9 & 96.9 & 97.0 \\
\hline $\mathrm{O}_{8}-\mathrm{H}_{17}$ & 96.9 & 96.9 & 96.9 & 97.0 \\
\hline $\mathrm{O}_{13}-\mathrm{H}_{21}$ & - & - & 97.2 & 97.2 \\
\hline $\mathrm{C}_{1}-\mathrm{H}_{14}$ & - & - & - & 108.5 \\
\hline $\mathrm{C}_{2}-\mathrm{H}_{15}$ & 108.7 & 108.4 & 108.7 & 108.6 \\
\hline $\mathrm{C}_{4}-\mathrm{H}_{16}$ & 108.3 & 108.5 & 108.3 & 108.7 \\
\hline $\mathrm{C}_{9}-\mathrm{H}_{19}$ & 108.9 & 109.0 & 108.9 & 108.9 \\
\hline $\mathrm{C}_{10}-\mathrm{H}_{20}$ & 108.5 & 108.5 & 108.4 & 108.5 \\
\hline $\mathrm{C}_{21}-\mathrm{H}_{23}$ & 109.5 & 109.5 & - & - \\
\hline $\mathrm{C}_{21}-\mathrm{H}_{24}$ & 109.5 & 109.5 & - & - \\
\hline $\mathrm{C}_{25}-\mathrm{H}_{26}$ & 109.4 & 109.4 & - & - \\
\hline $\mathrm{C}_{25}-\mathrm{H}_{27}$ & 109.4 & 109.4 & - & - \\
\hline $\mathrm{C}_{25}-\mathrm{H}_{28}$ & 109.4 & 109.4 & - & - \\
\hline \multicolumn{5}{|l|}{ Bond angles (degrees) } \\
\hline $\mathrm{C}_{2}-\mathrm{C}_{3}-\mathrm{C}_{4}$ & 119.2 & 119.1 & 119.2 & 118.1 \\
\hline $\mathrm{C}_{9}-\mathrm{C}_{3}-\mathrm{C}_{2}$ & 118.0 & 122.7 & 118.0 & 118.9 \\
\hline $\mathrm{C}_{10}-\mathrm{C}_{9}-\mathrm{C}_{3}$ & 128.0 & 128.0 & 128.0 & 128.2 \\
\hline $\mathrm{C}_{11}-\mathrm{C}_{10}-\mathrm{C}_{9}$ & 119.9 & 120.1 & 119.8 & 111.5 \\
\hline $\mathrm{C}_{10}-\mathrm{C}_{11}-\mathrm{O}_{13}$ & 110.7 & 110.7 & 111.5 & 111.5 \\
\hline $\mathrm{C}_{21}-\mathrm{O}_{13}-\mathrm{C}_{11}$ & 115.7 & 115.6 & - & - \\
\hline $\mathrm{C}_{25}-\mathrm{C}_{21}-\mathrm{O}_{13}$ & 107.5 & 107.5 & - & - \\
\hline $\mathrm{O}_{14}-\mathrm{C}_{1}-\mathrm{C}_{2}$ & 125.0 & 124.9 & 125.0 & - \\
\hline $\mathrm{O}_{7}-\mathrm{C}_{6}-\mathrm{C}_{1}$ & 122.4 & 122.2 & 122.4 & 120.3 \\
\hline $\mathrm{O}_{8}-\mathrm{C}_{5}-\mathrm{C}_{6}$ & 119.9 & 120.1 & 119.9 & 114.4 \\
\hline $\mathrm{O}_{12}-\mathrm{C}_{11}-\mathrm{O}_{13}$ & 123.2 & 123.2 & 122.0 & 121.9 \\
\hline $\mathrm{C}_{5}-\mathrm{O}_{8}-\mathrm{H}_{17}$ & 107.8 & 107.8 & 107.9 & 110.1 \\
\hline $\mathrm{C}_{6}-\mathrm{O}_{7}-\mathrm{H}_{18}$ & 108.0 & 108.0 & 108.0 & 107.8 \\
\hline $\mathrm{C}_{1}-\mathrm{O}_{14}-\mathrm{H}_{22}$ & 109.8 & 109.8 & 109.9 & - \\
\hline $\mathrm{C}_{11}-\mathrm{O}_{13}-\mathrm{H}_{21}$ & - & - & 105.6 & 105.5 \\
\hline $\mathrm{O}_{13}-\mathrm{C}_{21}-\mathrm{H}_{23}$ & 108.9 & 108.9 & - & - \\
\hline $\mathrm{C}_{21}-\mathrm{C}_{25}-\mathrm{H}_{26}$ & 110.9 & 110.9 & - & - \\
\hline $\mathrm{C}_{21}-\mathrm{C}_{25}-\mathrm{H}_{27}$ & 109.9 & 109.9 & - & - \\
\hline $\mathrm{C}_{3}-\mathrm{C}_{9}-\mathrm{H}_{19}$ & 116.0 & 116.1 & 115.9 & 116.0 \\
\hline $\mathrm{C}_{9}-\mathrm{C}_{10}-\mathrm{H}_{20}$ & 123.2 & 123.3 & 123.3 & 123.3 \\
\hline \multicolumn{5}{|c|}{ dihedral angles (degrees) } \\
\hline $\mathrm{C}_{3}-\mathrm{C}_{4}-\mathrm{C}_{5}-\mathrm{C}_{6}$ & 0.0 & 0.0 & 0.0 & 0.0 \\
\hline $\mathrm{C}_{9}-\mathrm{C}_{3}-\mathrm{C}_{4}-\mathrm{C}_{5}$ & 180.0 & 180.0 & -180.0 & 180.0 \\
\hline $\mathrm{C}_{10}-\mathrm{C}_{9}-\mathrm{C}_{3}-\mathrm{C}_{2}$ & 180.0 & 0.0 & -180.0 & 180.0 \\
\hline $\mathrm{C}_{11}-\mathrm{C}_{10}-\mathrm{C}_{9}-\mathrm{C}_{3}$ & 180.0 & 180.0 & 180.0 & 180.0 \\
\hline $\mathrm{O}_{12}-\mathrm{C}_{11}-\mathrm{C}_{10}-\mathrm{C}_{9}$ & 0.0 & 0.0 & 0.0 & 0.0 \\
\hline $\mathrm{O}_{13}-\mathrm{C}_{11}-\mathrm{C}_{10}-\mathrm{C}_{9}$ & 180.0 & -180.0 & -180.0 & 180.0 \\
\hline
\end{tabular}


Table 1 (continued)

\begin{tabular}{lllll}
\hline$\Delta E\left(\mathrm{kJmol}^{-1}\right) / \mu(\mathrm{D})^{\mathrm{a}}$ & tETHPPE 1 & cETHPPE 1 & tTHPPE $^{\mathrm{b}}$ & $\mathrm{CA}^{\mathrm{c}}$ \\
\cline { 2 - 5 } & $0 ; 2.6^{\mathrm{d}}$ & $0 ; 1.9$ & $0 ; 3.5$ & $0 ; 3.5$ \\
\hline $\mathrm{C}_{25}-\mathrm{C}_{21}-\mathrm{O}_{13}-\mathrm{C}_{11}$ & 180.0 & -180.0 & - & - \\
$\mathrm{O}_{12}-\mathrm{C}_{11}-\mathrm{O}_{13}-\mathrm{C}_{21}$ & 0.0 & 0.0 & - & - \\
$\mathrm{C}_{21}-\mathrm{O}_{13}-\mathrm{C}_{11}-\mathrm{C}_{10}$ & 180.0 & -180.0 & - & - \\
$\mathrm{H}_{15}-\mathrm{C}_{2}-\mathrm{C}_{3}-\mathrm{C}_{9}$ & 0.0 & 0.0 & 0.0 & 0.0 \\
$\mathrm{H}_{16}-\mathrm{C}_{4}-\mathrm{C}_{3}-\mathrm{C}_{2}$ & 180.0 & -180.0 & 180.0 & 180.0 \\
$\mathrm{H}_{18}-\mathrm{O}_{7}-\mathrm{C}_{6}-\mathrm{C}_{1}$ & 0.0 & 0.0 & 0.0 & 180.0 \\
$\mathrm{H}_{20}-\mathrm{C}_{10}-\mathrm{C}_{9}-\mathrm{C}_{3}$ & 0.0 & 0.00 & 0.0 & 0.0 \\
$\mathrm{H}_{21}-\mathrm{O}_{13}-\mathrm{C}_{11}-\mathrm{C}_{10}$ & - & - & 180.0 & 180.0 \\
$\mathrm{H}_{22}-\mathrm{O}_{14}-\mathrm{C}_{1}-\mathrm{C}_{2}$ & 0.0 & 0.0 & 0.0 & - \\
$\mathrm{H}_{23}-\mathrm{C}_{21}-\mathrm{O}_{13}-\mathrm{C}_{11}$ & 58.4 & 58.4 & - & - \\
$\mathrm{H}_{26}-\mathrm{C}_{25}-\mathrm{C}_{21}-\mathrm{O}_{13}$ & -60.2 & -60.2 & - \\
$\mathrm{H}_{28}-\mathrm{C}_{25}-\mathrm{C}_{21}-\mathrm{O}_{13}$ & 180.0 & 180.0 & - & - \\
\hline
\end{tabular}

Values for trans-THPPE and caffeic acid are included for comparison.

a Total dipole moment $1 D=1 / 3 \times 10^{-2} \mathrm{Cm}$.

b Most stable conformer [24].

c Most stable conformer [37].

${ }^{\mathrm{d}}$ Total value of energy for the most stable conformer of ETHPPE is -802.531196500 (in Hartree, $1 \mathrm{Hartree}^{2}=2625.5001 \mathrm{~kJ}^{\mathrm{mol}}{ }^{-1}$ ).

e Atoms are numbered according to Fig. 2.

species displaying different orientations of the ring $\mathrm{OH}^{\prime}$, the effect of the rotation around $\mathrm{C}_{9}-\mathrm{C}_{3}$ on the conformational energy is almost negligible: for the trans species, tETHPPE 7 $\left(\left(\mathrm{H}_{17} \mathrm{O}_{8} \mathrm{C}_{5} \mathrm{C}_{6}\right)=180^{\circ}, \quad\left(\mathrm{C}_{10} \mathrm{C}_{9} \mathrm{C}_{3} \mathrm{C}_{2}\right)=180^{\circ}, \quad \Delta E=\right.$ $\left.16.3 \mathrm{~kJ} \mathrm{~mol}^{-1}\right)$ vs tETHPPE $8 \quad\left(\left(\mathrm{H}_{17} \mathrm{O}_{8} \mathrm{C}_{5} \mathrm{C}_{6}\right)=180^{\circ}\right.$, $\left.\left(\mathrm{C}_{10} \mathrm{C}_{9} \mathrm{C}_{3} \mathrm{C}_{2}\right)=0^{\circ}, \Delta E=16.4 \mathrm{~kJ} \mathrm{~mol}^{-1}\right)$; for the cis isomer, cETHPPE $3\left(\left(\mathrm{H}_{17} \mathrm{O}_{8} \mathrm{C}_{5} \mathrm{C}_{6}\right)=180^{\circ},\left(\mathrm{C}_{10} \mathrm{C}_{9} \mathrm{C}_{3} \mathrm{C}_{2}\right)=0^{\circ}, \Delta E=\right.$ $\left.16.7 \mathrm{~kJ} \mathrm{~mol}^{-1}\right)$ vs cETHPPE $4 \quad\left(\left(\mathrm{H}_{17} \mathrm{O}_{8} \mathrm{C}_{5} \mathrm{C}_{6}\right)=180^{\circ}\right.$, $\left.\left(\mathrm{C}_{10} \mathrm{C}_{9} \mathrm{C}_{3} \mathrm{C}_{2}\right)=180^{\circ}, \Delta E=18.4 \mathrm{~kJ} \mathrm{~mol}^{-1}\right)$. Rotation about $\mathrm{C}_{11}-\mathrm{C}_{10}$, in turn, has a significant effect: tETHPPE 8 $\left(\left(\mathrm{H}_{17} \mathrm{O}_{8} \mathrm{C}_{5} \mathrm{C}_{6}\right)=180^{\circ}, \quad\left(\mathrm{O}_{13} \mathrm{C}_{11} \mathrm{C}_{10} \mathrm{C}_{9}\right)=180^{\circ}, \quad \Delta E=\right.$ $\left.16.4 \mathrm{~kJ} \mathrm{~mol}^{-1}\right)$ vs tETHPPE $9 \quad\left(\left(\mathrm{H}_{17} \mathrm{O}_{8} \mathrm{C}_{5} \mathrm{C}_{6}\right)=0^{\circ}\right.$, $\left.\left(\mathrm{O}_{13} \mathrm{C}_{11} \mathrm{C}_{10} \mathrm{C}_{9}\right)=0^{\circ}, \Delta E=20.7 \mathrm{~kJ} \mathrm{~mol}^{-1}\right)$.

Internal rotation around $\mathrm{O}_{13}-\mathrm{C}_{11}$-defining the relative position of the ester moiety - was found to be the most determinant factor for the overall stability of this kind of systems. Actually, the only two conformers with an ethyl Strans orientation were determined to be greatly unfavoured as compared to their $\mathrm{S}$-cis counterparts: tETHPPE 11 $\left(\left(\mathrm{C}_{10} \mathrm{C}_{9} \mathrm{C}_{3} \mathrm{C}_{2}\right)=0^{\circ}, \Delta E=33.9 \mathrm{~kJ} \mathrm{~mol}^{-1}\right)$ vs tETHPPE 1 $\left(\left(\mathrm{C}_{21} \mathrm{O}_{13} \mathrm{C}_{11} \mathrm{C}_{10}\right)=180^{\circ}, \quad \Delta E=0\right)$, and tETHPPE 12 $\left(\left(\mathrm{C}_{10} \mathrm{C}_{9} \mathrm{C}_{3} \mathrm{C}_{2}\right)=0^{\circ}, \Delta E=36.2 \mathrm{~kJ} \mathrm{~mol}^{-1}\right)$ vs tETHPPE 2 $\left(\left(\mathrm{C}_{21} \mathrm{O}_{13} \mathrm{C}_{11} \mathrm{C}_{10}\right)=180^{\circ}, \Delta E=0.6 \mathrm{~kJ} \mathrm{~mol}^{-1}\right)$ (Figs. 2(a) and 3(a)). These S-trans geometries are the highest energy ones obtained for ETHPPE, probably as a result of destabilising $\mathrm{H}_{20} \cdots \mathrm{H}_{26}$ and $\mathrm{H}_{20} \cdots \mathrm{H}_{27}$ repulsive interactions $\left(\mathrm{H}_{20} \cdots \mathrm{H}_{26} / \mathrm{H}_{27}\right.$ distances equal to $227 \mathrm{pm}$ ) (Fig. 3(a)). In fact, even the nonplanar cis isomer cETHPPE 6, displaying an S-cis ester group, is favoured relative to the trans conformers tETHPPE 11 and tETHPPE $12\left(\Delta E=20.7 \mathrm{vs} 33.9 \mathrm{~kJ} \mathrm{~mol}^{-1}\right.$ and $36.2 \mathrm{~kJ} \mathrm{~mol}^{-1}$, respectively, Fig. 2), possibly due to the formation of a stabilising intramolecular $\mathrm{H}_{15} \cdots \mathrm{O}_{13}$ close contact $\left(\mathrm{d}\left(\mathrm{H}_{15} \cdots\right.\right.$ $\left.\mathrm{O}_{13}\right)=211 \mathrm{pm}$, Fig. 3) which is not possible in tETHPPE 11 and tETHPPE 12.

Regarding the orientation of the ring hydroxyl groups, it was verified that an identical conformation of the three $\mathrm{OH}$ 's, coplanar with the ring, rendered the most stable conformers, since it leads to a minimisation of the steric repulsions between adjacent OH's and allows the formation of medium strength intramolecular $\mathrm{O} \cdots \mathrm{H}$ bonds $(\mathrm{O} \cdots \mathrm{H}$ distances between 217 and $219 \mathrm{pm}$, Fig. 3). In turn, those geometries where one of these hydroxyls has an opposite orientation relative to the other two, although still in-plane with the aromatic ring $\left(\left(\mathrm{H}_{17} \mathrm{O}_{8} \mathrm{C}_{5} \mathrm{C}_{6}\right)=180^{\circ}\right)$-tETHPPE 7, tETHPPE 8, tETHPPE 9, tETHPPE 10, cETHPPE 3 and CETHPPE 4-showed to be energetically unfavoured as compared to their counterparts with $\left(\mathrm{H}_{17} \mathrm{O}_{8} \mathrm{C}_{5} \mathrm{C}_{6}\right)=0^{\circ}$ (Figs. 2 and 3): for the trans isomerstETHPPE $1(\Delta E=0)$ vs tETHPPE $7\left(\Delta E=16.3 \mathrm{~kJ} \mathrm{~mol}^{-1}\right)$, tETHPPE $2\left(\Delta E=0.6 \mathrm{~kJ} \mathrm{~mol}^{-1}\right)$ vs tETHPPE $8(\Delta E=$ $\left.16.4 \mathrm{~kJ} \mathrm{~mol}^{-1}\right)$, tETHPPE $3\left(\Delta E=4.1 \mathrm{~kJ} \mathrm{~mol}^{-1}\right)$ vs tETHPPE $9\left(\Delta E=20.7 \mathrm{~kJ} \mathrm{~mol}^{-1}\right)$, and tETHPPE $4\left(\Delta E=5.2 \mathrm{~kJ} \mathrm{~mol}^{-1}\right)$ vs tETHPPE $102\left(\Delta E=21.0 \mathrm{~kJ} \mathrm{~mol}^{-1}\right)$; for the cis speciescETHPPE $1(\Delta E=0)$ vs CETHPPE $3\left(\Delta E=16.7 \mathrm{~kJ} \mathrm{~mol}^{-1}\right)$, and CETHPPE $2\left(\Delta E=9.4 \mathrm{~kJ} \mathrm{~mol}^{-1}\right)$ vs CETHPPE $4(\Delta E=$ $18.4 \mathrm{~kJ} \mathrm{~mol}^{-1}$ ). In addition, the relative orientation of the central $\mathrm{OH}$ (as long as it remains in-plane) is practically irrelevant-tETHPPE $7\left(\Delta E=16.3 \mathrm{~kJ} \mathrm{~mol}^{-1}\right)$ vs tETHPPE 8 $\left(\Delta E=16.4 \mathrm{~kJ} \mathrm{~mol}^{-1}\right)$. In turn, an out-of-plane hydroxyl group, quasi-perpendicular relative to the benzene ring $\left(\left(\mathrm{H}_{18} \mathrm{O}_{7} \mathrm{C}_{6} \mathrm{C}_{1}\right)\right.$ ca. 90 ${ }^{\circ}$-tETHPPE 5, tETHPPE 6 and cETHPPE 5-was verified to be responsible for a clear destabilisation (Fig. 2), along with an orientation of the two neighbouring $\mathrm{H}_{22}$ and $\mathrm{H}_{17}$ atoms towards the central $\mathrm{O}_{7}\left(\mathrm{~d}\left(\mathrm{H}_{17} / \mathrm{H}_{22} \cdots \mathrm{O}_{7}\right)\right.$ ca. $222 \mathrm{pm}$, Fig. 3): regarding the trans species-tETHPPE $1(\Delta E=0)$ vs tETHPPE $5\left(\left(\mathrm{H}_{18} \mathrm{O}_{7} \mathrm{C}_{6} \mathrm{C}_{1}\right)=91.9^{\circ}, \Delta E=10.6 \mathrm{~kJ} \mathrm{~mol}^{-1}\right)$, and tETHPPE $3\left(\Delta E=4.1 \mathrm{~kJ} \mathrm{~mol}^{-1}\right) \quad$ vs tETHPPE 6 $\left(\left(\mathrm{H}_{18} \mathrm{O}_{7} \mathrm{C}_{6} \mathrm{C}_{1}\right)=91.3^{\circ}, \Delta E=14.8 \mathrm{~kJ} \mathrm{~mol}^{-1}\right)$; for the cis isomers-cETHPPE $1(\Delta E=0)$ vs cETHPPE $5\left(\left(\mathrm{H}_{18} \mathrm{O}_{7} \mathrm{C}_{6} \mathrm{C}_{1}\right)=\right.$ $\left.94.0^{\circ}, \Delta E=19.3 \mathrm{~kJ} \mathrm{~mol}^{-1}\right)$. As expected, no minimum energy geometries were obtained when any two ring hydroxyls were directed towards each other. This is in accordance with the results previously reported for the corresponding acid THPPE, 
Table 2

Experimental (solid state) and calculated (B3LYP/6-31G**) Raman wavenumbers $\left(\mathrm{cm}^{-1}\right)$ for the most stable conformers of trans-ethyl 3-(3,4,5-trihydroxyphenyl)2-propenoate (ETHPPE) and diethyl 2-(3,4,5-trihydroxyphenylmethylene)malonate (E2THPPE)

\begin{tabular}{|c|c|c|c|c|c|c|c|}
\hline \multicolumn{4}{|c|}{ Experimental } & \multicolumn{3}{|l|}{ Calculated $^{\mathrm{a}}$} & \multirow[t]{2}{*}{ Approximate description ${ }^{\mathrm{b}}$} \\
\hline THPPE $^{\mathrm{c}}$ & ETHPPE & E2THPPE & $\mathrm{tTHPPE}^{\mathrm{d}}$ & $\begin{array}{l}\text { tETHPPE } 1 \\
(44 \%)^{d}\end{array}$ & $\begin{array}{l}\text { tETHPPE } 2 \\
(37 \%)^{d}\end{array}$ & $\begin{array}{l}\text { E2THPPE } 1 \\
(21 \%)^{\mathrm{d}}\end{array}$ & \\
\hline 3412 & 3399 & 3474 & $3696(72 ; 117)$ & $3695(72 ; 119)$ & $3694(61 ; 95)$ & $3688(62 ; 84)$ & $\nu\left(\mathrm{O}^{14} \mathrm{H}\right)$ \\
\hline 3395 & & & & & & & $2 \times \nu(\mathrm{C}=\mathrm{O})$ \\
\hline 3363 & 3369 & 3426 & $3648(140 ; 180)$ & $3646(145 ; 191)$ & $3646(132 ; 169)$ & $3642(118 ; 162)$ & $\nu\left(\mathrm{O}^{7} \mathrm{H}\right)$ \\
\hline 3350 & 3342 & 3414 & $3637(99 ; 100)$ & $3636(101 ; 103)$ & $3637(98 ; 107)$ & $3639(118 ; 139)$ & $\nu\left(\mathrm{O}^{8} \mathrm{H}\right)$ \\
\hline \multirow[t]{2}{*}{3319} & & & $3619(87 ; 198)$ & & & & $\nu\left(\mathrm{O}^{13} \mathrm{H}\right)$ \\
\hline & 3321 & 3343 & & & & & $2 \times \nu(\mathrm{C}=\mathrm{O})$ \\
\hline 3277 & 3210 & 3205 & & & & & $2 \times \nu(\mathrm{C}=\mathrm{C})$ \\
\hline 3124 & 3064 & & $3101(3 ; 55)$ & $3101(3 ; 47)$ & $3090(2 ; 65)$ & & $\nu_{\mathrm{s}}\left(\mathrm{C}^{2} \mathrm{H}+\mathrm{C}^{10} \mathrm{H}\right)$ \\
\hline 3080 & 3033 & & $3089(4 ; 14)$ & $3086(5 ; 15)$ & $3082(11 ; 41)$ & & $\nu_{\text {as }}\left(\mathrm{C}^{2} \mathrm{H}+\mathrm{C}^{10} \mathrm{H}\right)$ \\
\hline \multirow[t]{4}{*}{3067} & & 3104 & $3059(8 ; 71)$ & & & $3099(28 ; 41)$ & $\nu\left(\mathrm{C}^{2} \mathrm{H}\right)$ \\
\hline & & & & & & $3091(1 ; 62)$ & $\nu\left(\mathrm{C}^{4} \mathrm{H}\right)$ \\
\hline & & & & $3056(9 ; 74)$ & $3065(4 ; 20)$ & & $\nu_{\mathrm{s}}\left(\mathrm{C}^{4} \mathrm{H}+\mathrm{C}^{9} \mathrm{H}\right)$ \\
\hline & 3009 & & & $3044(1 ; 22)$ & $3049(1 ; 33)$ & & $\nu_{\text {as }}\left(\mathrm{C}^{4} \mathrm{H}+\mathrm{C}^{9} \mathrm{H}\right)$ \\
\hline \multirow{16}{*}{$\begin{array}{l}3053 \\
3020\end{array}$} & & 3046 & $3045(1 ; 27)$ & & & $3042(0 ; 36)$ & $\nu\left(\mathrm{C}^{9} \mathrm{H}\right)$ \\
\hline & & & & & & & $(1375+1640) \mathrm{cm}^{-1}$ \\
\hline & 2988 & & & $3015(36 ; 30)$ & $3015(37 ; 29)$ & $3020(29 ; 22)$ & $\nu_{\text {as }}\left(\mathrm{CH}_{2}\right)+\nu_{\text {as }}\left(\mathrm{CH}_{3}\right)$ \\
\hline & & & & & & $3018(36 ; 20)$ & $\nu_{\text {as }}\left(\mathrm{CH}_{2}\right)+\nu_{\text {as }}\left(\mathrm{CH}_{3}\right)$ \\
\hline & 2965 & 3004 & & $3008(28 ; 124)$ & $3008(28 ; 125)$ & $3011(23 ; 89)$ & $\nu_{\text {as }}\left(\mathrm{CH}_{3}\right)$ \\
\hline & & 2984 & & & & $3010(29 ; 126)$ & $\nu_{\text {as }}\left(\mathrm{CH}_{3}\right)$ \\
\hline & 2951 & 2977 & & $2979(11 ; 84)$ & $2980(10 ; 85)$ & $2986(8 ; 80)$ & $\nu_{\mathrm{as}}\left(\mathrm{CH}_{2}\right)+\nu_{\mathrm{as}}\left(\mathrm{CH}_{3}\right)$ \\
\hline & & & & & & $2986(8 ; 70)$ & $\nu_{\text {as }}\left(\mathrm{CH}_{2}\right)$ \\
\hline & 2942 & 2965 & & $2942(21 ; 128)$ & $2942(21 ; 123)$ & $2947(18 ; 117)$ & $\nu_{\mathrm{s}}\left(\mathrm{CH}_{2}\right)$ \\
\hline & & 2943 & & & & & $\left(1608+1325 \mathrm{~cm}^{-1}\right)$ FR $\nu_{\text {as }}(\mathrm{CH})$ \\
\hline & & & & & & $2946(18 ; 74)$ & $\nu_{\mathrm{s}}\left(\mathrm{CH}_{2}\right)$ \\
\hline & 2931 & 2934 & & $2937(21 ; 151)$ & $2936(21 ; 154)$ & $2939(17 ; 122)$ & $\nu_{\mathrm{s}}\left(\mathrm{CH}_{3}\right)$ \\
\hline & 2925 & & & & & & $\left(1601+1359 \mathrm{~cm}^{-1}\right)$ FR $\nu_{\text {as }}\left(\mathrm{CH}_{3}\right)$ \\
\hline & & & & & & $2938(18 ; 150)$ & $\nu_{\mathrm{s}}\left(\mathrm{CH}_{3}\right)$ \\
\hline & 2898 & & & & & & $(1601+1281) \mathrm{cm}^{-1}$ \\
\hline & & 1717 & & & & $1727(112 ; 39)$ & $\nu_{\mathrm{iph}}(\mathrm{CC}=\mathrm{O})$ \\
\hline \multirow[t]{4}{*}{1640} & 1660 & & $1739(258 ; 85)$ & $1719(183 ; 64)$ & $1722(188 ; 72)$ & & $\nu(\mathrm{CC}=\mathrm{O})$ \\
\hline & & 1676 & & & & $1712(23 ; 282)$ & $\nu_{\mathrm{oph}}(\mathrm{CC}=\mathrm{O})$ \\
\hline & & & $1634(218 ; 659)$ & & & & $\nu\left(\mathrm{C}^{9} \mathrm{C}=\mathrm{C}^{10}\right)$ \\
\hline & & 1645 & & $1634(198 ; 962)$ & $1632(223 ; 1049)$ & $1614(44 ; 42)$ & $\nu\left(\mathrm{C}^{9} \mathrm{C}=\mathrm{C}^{10}\right)+\nu(\mathrm{CC})_{\text {ring }}$ \\
\hline 1612 & 1601 & 1625 & $1601(157 ; 1606)$ & $1602(141 ; 2059)$ & $1605(69 ; 704)$ & $1602(123 ; 361)$ & $\nu(\mathrm{CC})_{\text {ring }}+\nu\left(\mathrm{C}^{9} \mathrm{C}=\mathrm{C}^{10}\right)$ \\
\hline 1583 & & 1608 & $1599(157 ; 1606)$ & $1599(132 ; 22)$ & $1597(181 ; 1252)$ & $1584(280 ; 2437)$ & $\nu(\mathrm{CC})_{\text {ring }}$ \\
\hline \multirow[t]{3}{*}{1538} & 1511 & 1536 & $1517(133 ; 18)$ & $1517(236 ; 2)$ & $1518(213 ; 1)$ & $1518(265 ; 16)$ & $\nu(\mathrm{CC})_{\text {ring }}$ \\
\hline & 1475 & 1465 & & $1473(5 ; 5)$ & $1474(6 ; 6)$ & $1473(4 ; 3)$ & $\delta\left(\mathrm{CH}_{2}\right)($ sciss. $)+\delta_{\mathrm{as}}\left(\mathrm{CH}_{3}\right)$ \\
\hline & & & & & & $1471(5 ; 1)$ & $\delta\left(\mathrm{CH}_{2}\right)$ (sciss. $)+\delta_{\text {as }}\left(\mathrm{CH}_{3}\right)$ \\
\hline \multirow[t]{9}{*}{1453} & 1424 & & $1459(232 ; 3)$ & $1459(19 ; 126)$ & $1455(61 ; 19)$ & & $\nu(\mathrm{CC})_{\text {ring }}+\delta\left(\mathrm{O}^{8} \mathrm{H}\right)$ \\
\hline & & & & $1454(3 ; 22)$ & $1454(20 ; 24)$ & $1455(1 ; 12)$ & $\delta\left(\mathrm{CH}_{2}\right)($ sciss. $)+\delta_{\mathrm{as}}\left(\mathrm{CH}_{3}\right)$ \\
\hline & & & & & & $1454(77 ; 28)$ & $\delta\left(\mathrm{O}^{8} \mathrm{H}\right)+\delta_{\text {as }}\left(\mathrm{CH}_{3}\right)$ \\
\hline & & & & & & $1452(12 ; 27)$ & $\delta_{\mathrm{as}}\left(\mathrm{CH}_{3}\right)+\delta\left(\mathrm{CH}_{2}\right)$ (sciss. $)$ \\
\hline & 1386 & 1403 & & $1443(5 ; 25)$ & $1443(5 ; 25)$ & $1443(4 ; 31)$ & $\delta_{\text {as }}\left(\mathrm{CH}_{3}\right)$ \\
\hline & & 1388 & & & & $1442(6 ; 14)$ & $\delta_{\text {as }}\left(\mathrm{CH}_{3}\right)$ \\
\hline & & & & & & $1388(8 ; 16)$ & $\delta_{\mathrm{s}}\left(\mathrm{CH}_{3}\right)+\omega\left(\mathrm{CH}_{2}\right)+\delta\left(\mathrm{C}^{9} \mathrm{H}\right)$ \\
\hline & 1374 & & & $1384(5 ; 9)$ & $1384(0 ; 15)$ & $1383(7 ; 6)$ & $\delta_{\mathrm{s}}\left(\mathrm{CH}_{3}\right)+\omega\left(\mathrm{CH}_{2}\right)$ \\
\hline & & & & & & $1375(51 ; 10)$ & $\delta_{\mathrm{s}}\left(\mathrm{CH}_{3}\right)+\delta\left(\mathrm{C}^{9} \mathrm{H}\right)$ \\
\hline 1374 & 1358 & 1368 & $1375(22 ; 96)$ & $1366(7 ; 119)$ & $1372(58 ; 135)$ & $1366(117 ; 279)$ & $\begin{array}{l}\nu(\mathrm{CC})_{\text {ring }}+\delta\left(\mathrm{O}^{7} \mathrm{H}\right)+\delta\left(\mathrm{O}^{8} \mathrm{H}\right)+\delta_{\mathrm{s}} \\
\left(\mathrm{CH}_{3}\right)+\nu\left(\mathrm{C}^{1} \mathrm{O}\right)\end{array}$ \\
\hline \multirow[t]{5}{*}{1355} & & & $1362(1 ; 99)$ & $1360(20 ; 23)$ & $1354(149 ; 168)$ & & $\begin{array}{l}\nu(\mathrm{CC})_{\text {ring }}+\delta\left(\mathrm{O}^{7} \mathrm{H}\right)+\delta\left(\mathrm{C}^{9} \mathrm{H}\right)+\delta_{\mathrm{s}} \\
\left(\mathrm{CH}_{3}\right)+\omega\left(\mathrm{CH}_{2}\right)\end{array}$ \\
\hline & & 1325 & & & & $1350(38 ; 21)$ & $\delta\left(\mathrm{O}^{7} \mathrm{H}\right)+\delta_{\mathrm{s}}\left(\mathrm{CH}_{3}\right)+\omega\left(\mathrm{CH}_{2}\right)$ \\
\hline & 1316 & & & $1345(13 ; 14)$ & $1348(4 ; 1)$ & $1348(10 ; 13)$ & $\omega\left(\mathrm{CH}_{2}\right)+\delta_{\mathrm{s}}\left(\mathrm{CH}_{3}\right)$ \\
\hline & & & & & & $1337(32 ; 11)$ & $\begin{array}{l}\omega\left(\mathrm{CH}_{2}\right)+\delta_{\mathrm{s}}\left(\mathrm{CH}_{3}\right)+\delta\left(\mathrm{O}^{7} \mathrm{H}\right)+\delta \\
\left(\mathrm{O}^{8} \mathrm{H}\right)+\delta\left(\mathrm{C}^{9} \mathrm{H}\right)\end{array}$ \\
\hline & 1281 & & $1329(33 ; 39)$ & $1309(211 ; 129)$ & $1313(40 ; 13)$ & & $\delta(\mathrm{CH})+\delta(\mathrm{OH})$ \\
\hline 1307 & & & $1306(157 ; 2)$ & & & $1298(147 ; 17)$ & $\delta(\mathrm{OH})+\delta\left(\mathrm{C}^{9} \mathrm{H}\right)+\delta_{\mathrm{as}}\left(\mathrm{C}^{2} \mathrm{H}+\mathrm{C}^{4} \mathrm{H}\right)$ \\
\hline 1289 & & & $1292(428 ; 140)$ & $1295(43 ; 9)$ & $1294(85 ; 21)$ & & $\delta_{\mathrm{as}}\left(\mathrm{C}^{9} \mathrm{H}+\mathrm{C}^{10} \mathrm{H}\right)+\delta(\mathrm{OH})$ \\
\hline
\end{tabular}


Table 2 (continued)

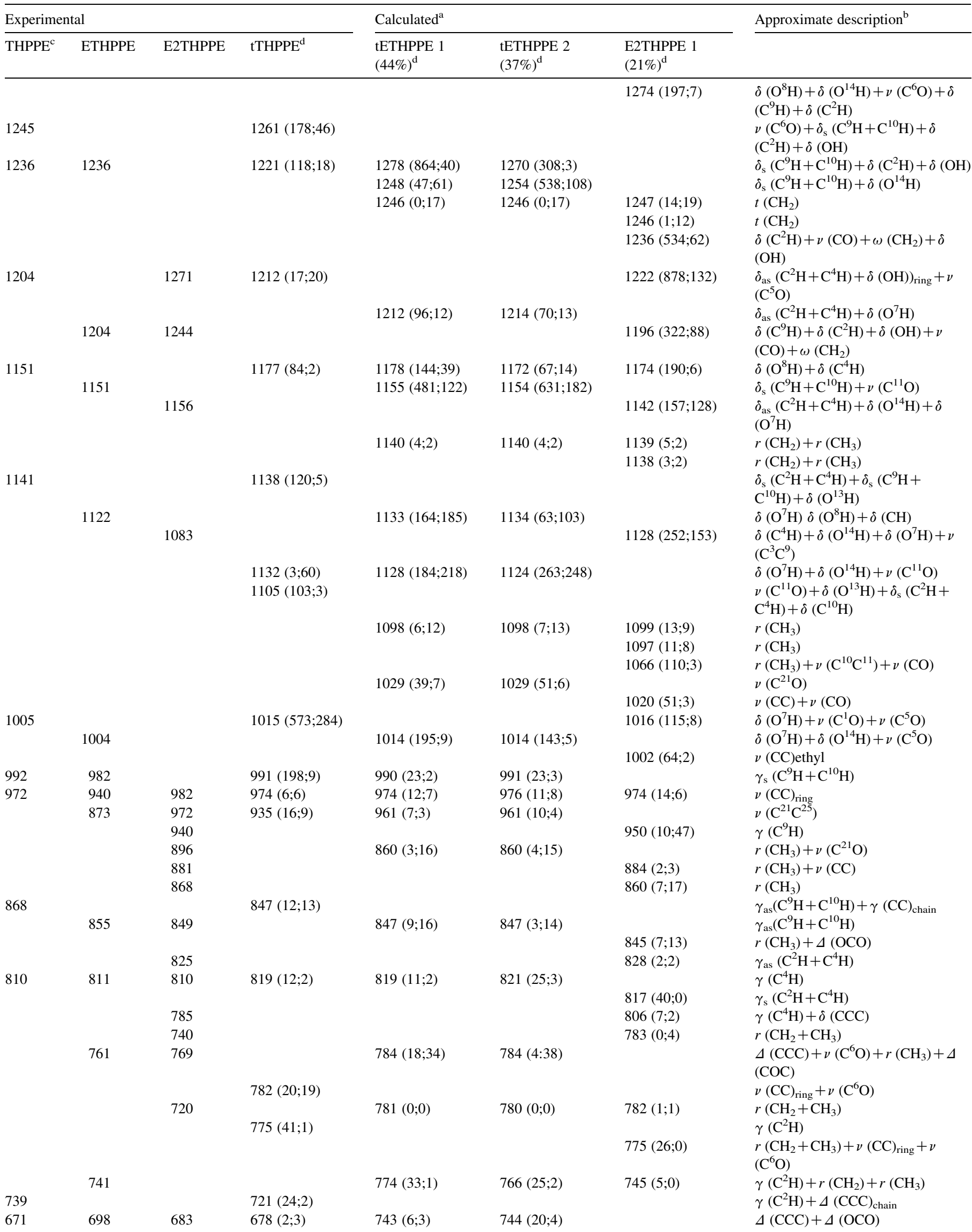


Table 2 (continued)

\begin{tabular}{|c|c|c|c|c|c|c|c|}
\hline \multicolumn{4}{|c|}{ Experimental } & \multicolumn{3}{|l|}{ Calculated $^{\mathrm{a}}$} & \multirow[t]{2}{*}{ Approximate description $^{\mathrm{b}}$} \\
\hline THPPE $^{\mathrm{c}}$ & ETHPPE & E2THPPE & $\mathrm{tTHPPE}^{\mathrm{d}}$ & $\begin{array}{l}\text { tETHPPE } 1 \\
(44 \%)^{d}\end{array}$ & $\begin{array}{l}\text { tETHPPE } 2 \\
(37 \%)^{\mathrm{d}}\end{array}$ & $\begin{array}{l}\text { E2THPPE } 1 \\
(21 \%)^{\mathrm{d}}\end{array}$ & \\
\hline & & & & & & $718(26 ; 16)$ & $\Gamma(\mathrm{OCO})$ \\
\hline & 671 & & & $707(2 ; 0)$ & $707(3 ; 0)$ & & $\Gamma(\mathrm{CCC})$ \\
\hline & & 670 & & & & $653(38 ; 3)$ & $\Delta(\mathrm{CCC})$ \\
\hline & 647 & 662 & & $636(0 ; 0)$ & $636(0 ; 0)$ & $639(0 ; 1)$ & $\Gamma(\mathrm{CCC})_{\text {ring }}$ \\
\hline & 623 & & & $623(1 ; 8)$ & $625(58 ; 2)$ & & $\Delta(\mathrm{CCC})+\Delta(\mathrm{CCO})$ \\
\hline & & 622 & & & & $617(11 ; 1)$ & $\Delta(\mathrm{CCC})_{\text {ring }}$ \\
\hline & & 601 & $637(2 ; 0)$ & & & $595(1 ; 22)$ & $\Gamma(\mathrm{CCC})_{\text {ring }}$ \\
\hline 624 & 594 & & $616(11 ; 3)$ & $602(43 ; 5)$ & $601(2 ; 13)$ & & $\Delta(\mathrm{CCC})+\Delta(\mathrm{CCO})$ \\
\hline \multirow[t]{2}{*}{605} & 582 & 575 & $608(61 ; 6)$ & $583(2 ; 1)$ & $581(2 ; 0)$ & $517(2 ; 14)$ & $\Gamma(\mathrm{CCC})+\gamma\left(\mathrm{O}^{13} \mathrm{H}\right)$ \\
\hline & & 554 & & & & $557(13 ; 10)$ & $\Delta(\mathrm{CCC})_{\text {ring }}+\Gamma(\mathrm{CCC})_{\text {chain }}$ \\
\hline \multirow[t]{2}{*}{589} & & & $581(82 ; 12)$ & & & & $\Delta(\mathrm{CCC})+\Delta(\mathrm{OCO})$ \\
\hline & & & $558(26 ; 2)$ & & & & $\Gamma(\mathrm{CCC})+\gamma\left(\mathrm{O}^{13} \mathrm{H}\right)$ \\
\hline 541 & & & $546(8 ; 0)$ & $548(0 ; 0)$ & $548(1 ; 0)$ & $550(0 ; 0)$ & $\Gamma(\mathrm{CCC})_{\text {ring }}$ \\
\hline 521 & 541 & & $522(0 ; 4)$ & $522(1 ; 4)$ & $520(0 ; 4)$ & & $\Delta(\mathrm{CCC})_{\text {ring }}$ \\
\hline \multirow[t]{2}{*}{484} & & 483 & $489(25 ; 6)$ & & & $490(11 ; 4)$ & $\Delta(\mathrm{CCC})$ \\
\hline & 501 & 449 & & $486(9 ; 6)$ & $486(11 ; 5)$ & $481(1 ; 8)$ & $\Delta(\mathrm{CCC})_{\text {ring }}$ \\
\hline 452 & 480 & 435 & $454(11 ; 3)$ & $453(15 ; 6)$ & $453(1 ; 4)$ & $420(5 ; 4)$ & $\Delta(\mathrm{CCC})_{\text {chain }}+\Delta(\mathrm{CCO})$ \\
\hline \multirow[t]{2}{*}{434} & 394 & 421 & $426(51 ; 1)$ & $421(47 ; 1)$ & $420(50 ; 2)$ & $419(27 ; 1)$ & $\gamma\left(\mathrm{O}^{8} \mathrm{H}\right)+\gamma\left(\mathrm{O}^{7} \mathrm{H}\right)$ \\
\hline & 352 & 405 & & $388(24 ; 4)$ & $387(17 ; 3)$ & $386(26 ; 8)$ & $\Gamma(\mathrm{CCC})+\Gamma(\mathrm{CCO})$ \\
\hline \multirow[t]{3}{*}{380} & & & $387(20 ; 5)$ & & & & $\Gamma(\mathrm{CCC})$ \\
\hline & 331 & 391 & & $379(11 ; 1)$ & $380(7 ; 1)$ & $383(10 ; 3)$ & $\Delta(\mathrm{CCO})$ \\
\hline & & 359 & & & & $376(72 ; 2)$ & $\begin{array}{l}\gamma\left(\mathrm{O}^{14} \mathrm{H}\right)+\gamma\left(\mathrm{O}^{8} \mathrm{H}\right)+\gamma\left(\mathrm{O}^{7} \mathrm{H}\right)+\Delta \\
(\mathrm{CCO})\end{array}$ \\
\hline \multirow[t]{3}{*}{352} & 317 & 346 & $357(104 ; 2)$ & $357(108 ; 3)$ & $357(110 ; 3)$ & $371(54 ; 2)$ & $\gamma\left(\mathrm{O}^{14} \mathrm{H}\right)+\gamma\left(\mathrm{O}^{8} \mathrm{H}\right)+\gamma\left(\mathrm{O}^{7} \mathrm{H}\right)$ \\
\hline & & 335 & & & & $316(10 ; 3)$ & $\gamma\left(\mathrm{O}^{8} \mathrm{H}\right)+\gamma(\mathrm{CC})$ \\
\hline & & & & & & $314(22 ; 3)$ & $\gamma\left(\mathrm{O}^{8} \mathrm{H}\right)+\gamma(\mathrm{CC})$ \\
\hline 323 & 304 & & $316(10 ; 1)$ & $313(16 ; 1)$ & $314(10 ; 1)$ & & $\Delta\left(\mathrm{C}^{1} \mathrm{OH}\right)+\Delta\left(\mathrm{C}^{5} \mathrm{OH}\right)$ \\
\hline \multirow[t]{3}{*}{290} & & 306 & $298(2 ; 0)$ & $298(0 ; 1)$ & $298(7 ; 1)$ & $295(3 ; 2)$ & $\Delta\left(\mathrm{C}^{6} \mathrm{OH}\right)$ \\
\hline & & 285 & & & & $286(12 ; 1)$ & $\tau\left(\mathrm{CH}_{3}\right)$ \\
\hline & 273 & & & $286(5 ; 1)$ & $285(10 ; 1)$ & & $\Gamma(\mathrm{CCC})+\Gamma(\mathrm{CCO})$ \\
\hline \multirow[t]{5}{*}{264} & & & $276(2 ; 4)$ & & & & $\Delta\left(\mathrm{C}^{1} \mathrm{OH}\right)+\Delta\left(\mathrm{C}^{11} \mathrm{OH}\right)$ \\
\hline & & & & & & $278(2 ; 0)$ & $\tau\left(\mathrm{CH}_{3}\right)+\Gamma(\mathrm{CCO})$ \\
\hline & & & & $275(0 ; 0)$ & $276(0 ; 0)$ & & $\tau\left(\mathrm{CH}_{3}\right)$ \\
\hline & & 268 & $265(5 ; 0)$ & & & $262(20 ; 2)$ & $\tau\left(\mathrm{CH}_{3}\right)+\Gamma(\mathrm{CCC})+\gamma\left(\mathrm{O}^{14} \mathrm{H}\right)$ \\
\hline & 262 & 261 & & $263(3 ; 0)$ & $266(7 ; 0)$ & $258(23 ; 2)$ & $\tau\left(\mathrm{CH}_{3}\right)+\Gamma(\mathrm{CCC})$ \\
\hline \multirow[t]{7}{*}{241} & 229 & 244 & $251(7 ; 1)$ & $251(4 ; 1)$ & $250(2 ; 3)$ & $256(54 ; 2)$ & $\Gamma(\mathrm{CCC})+\tau\left(\mathrm{CH}_{3}\right)+\gamma\left(\mathrm{O}^{14} \mathrm{H}\right)$ \\
\hline & & 226 & & & & $244(80 ; 2)$ & $\tau\left(\mathrm{CH}_{3}\right)+\gamma\left(\mathrm{O}^{14} \mathrm{H}\right)+\gamma\left(\mathrm{O}^{7} \mathrm{H}\right)$ \\
\hline & & & $213(0 ; 3)$ & & & & $\Delta(\mathrm{CCC})_{\text {chain }}$ \\
\hline & 202 & & & $209(0 ; 4)$ & $214(182 ; 3)$ & & $\Delta(\mathrm{CCC})+\Delta(\mathrm{CCO})$ \\
\hline & 191 & & $209(179 ; 5)$ & $208(184 ; 4)$ & $204(0 ; 4)$ & & $\gamma\left(\mathrm{O}^{14} \mathrm{H}\right)+\gamma\left(\mathrm{O}^{7} \mathrm{H}\right)$ \\
\hline & & 188 & & & & $204(2 ; 3)$ & $\tau\left(\mathrm{CH}_{3}\right)+\Delta(\mathrm{CCC})$ \\
\hline & 177 & 176 & & $189(0 ; 3)$ & $188(0 ; 3)$ & $189(3 ; 1)$ & $\tau\left(\mathrm{CH}_{3}\right)+\Gamma(\mathrm{CCC})$ \\
\hline \multirow[t]{4}{*}{179} & & 163 & $162(0 ; 1)$ & $158(0 ; 0)$ & $158(0 ; 0)$ & $159(0 ; 1)$ & $\Gamma(\mathrm{CCO})+\Gamma(\mathrm{CCC})$ \\
\hline & & & & & & $154(0 ; 0)$ & $\Gamma(\mathrm{CCC})$ \\
\hline & 141 & 139 & $137(1 ; 1)$ & $157(2 ; 1)$ & $158(2 ; 1)$ & $143(2 ; 1)$ & $\Delta(\mathrm{CCC})+\Delta(\mathrm{CCO})$ \\
\hline & & & & & & $109(0 ; 2)$ & $\Gamma(\mathrm{CCC})+\Gamma(\mathrm{CCO})$ \\
\hline \multirow[t]{9}{*}{127} & & & & $94(1 ; 0)$ & $94(1 ; 0)$ & $98(1 ; 1)$ & $\Gamma(\mathrm{CCC})+\Gamma(\mathrm{CCO})$ \\
\hline & & & $82(1 ; 0)$ & & & $82(0 ; 1)$ & Skeletal modes \\
\hline & & & & & & $70(0 ; 0)$ & Skeletal modes \\
\hline & & 111 & $68(0 ; 0)$ & $60(0 ; 1)$ & $59(0 ; 1)$ & $61(0 ; 0)$ & Skeletal modes \\
\hline & & & & $56(0 ; 1)$ & $55(0 ; 1)$ & $56(0 ; 0)$ & Skeletal modes \\
\hline & & & & $55(1 ; 0)$ & $55(0 ; 0)$ & & Skeletal modes \\
\hline & & & & & & $35(0 ; 0)$ & Skeletal modes \\
\hline & & & $34(2 ; 0)$ & $22(2 ; 1)$ & $21(0 ; 1)$ & $26(2 ; 2)$ & Skeletal modes \\
\hline & & & & & & $18(0 ; 4)$ & Skeletal modes \\
\hline
\end{tabular}

Values for trans-THPPE are included for comparison. $\delta$, in-plane deformation; $t$, twisting; $r$, rocking; $\omega$, wagging; sciss., scissoring; $\gamma$, out-of-plane deformation; $\Delta$, in-plane deformation of skeleton atoms; $\Gamma$, out-of-plane deformation of skeleton atoms; iph, in-phase; oph, out-of-phase; FR, Fermi Ressonance.

${ }^{\mathrm{a}}$ Wavenumbers above $400 \mathrm{~cm}^{-1}$ are scaled by a factor of 0.9614 [32]. (IR intensities in $\mathrm{km} \mathrm{mol}^{-1}$; Raman scattering activities in $\AA$ amu).

b Atoms are numbered according to Fig. 2.

${ }^{c}$ [24]; the calculated wavenumbers are the ones obtained for the most stable conformer.

${ }^{\mathrm{d}}$ Relative population $\left(\right.$ at $\left.25^{\circ} \mathrm{C}\right)$. 


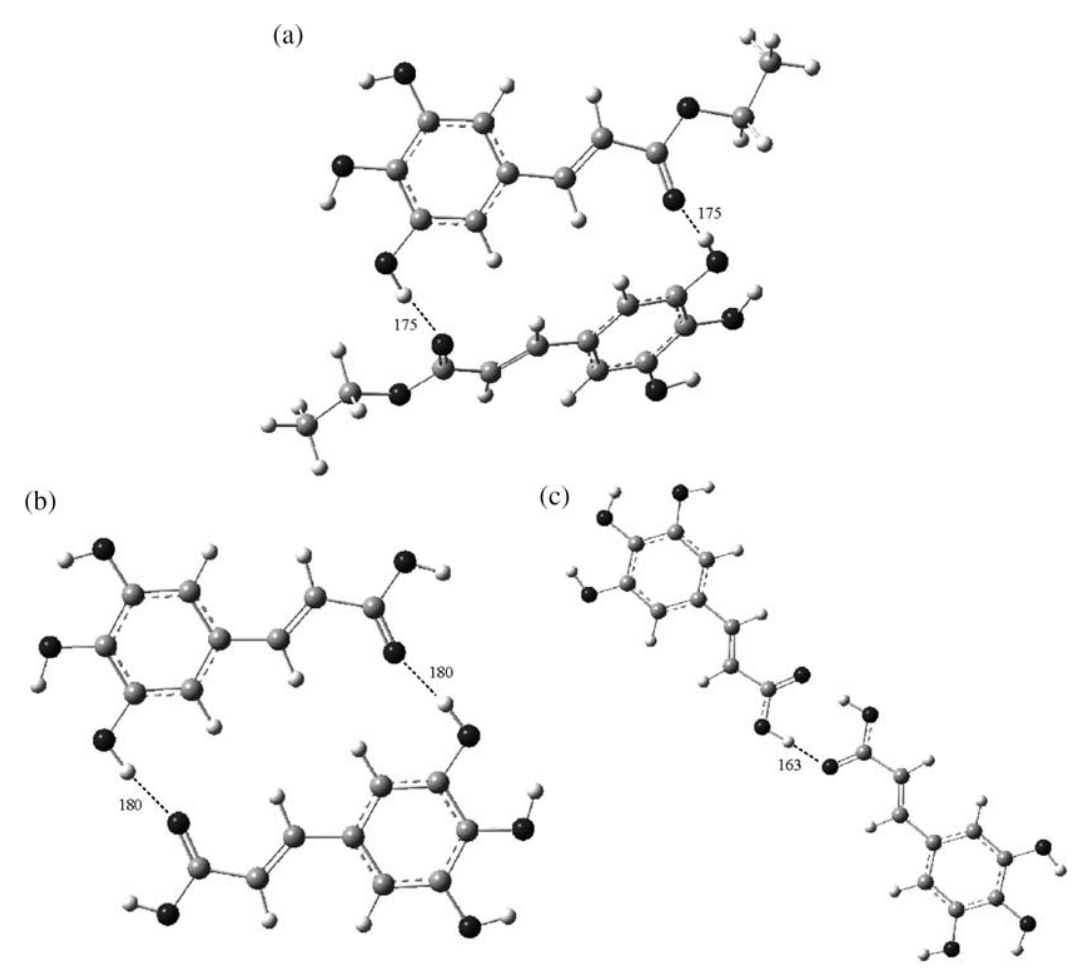

Fig. 4. Representation of calculated dimeric structures for: (a) trans-ethyl 3-(3,4,5-trihydroxyphenyl)-2-propenoate (ETHPPE); (b) and (c) trans-3-(3,4,5-trihydroxyphenyl)-2-propenoic acid (THPPE). (B3LYP/6-31G** level of calculation. Distances are represented in pm).

for which only one such geometry was calculated as a highly unfavoured conformer [24].

Table 1 comprises the calculated optimised geometries for the lowest energy trans- and cis-ETHPPE geometries (values for the other conformers are available from the authors upon request). These structural parameters do not deviate much from the X-ray values found in the literature for the analogous dihydroxylated cinnamic acid (known as caffeic acid) [35], and also agree well with the calculated values previously reported for trans-caffeic acid [36,37] and trans-THPPE [24]. The smaller number of conformers calculated for ETHPPE relative to THPPE (18 vs 21) is a result of destabilising steric hindrances due to the presence of the ethyl ester group, quite bulkier than the carboxylic $\mathrm{OH}$ (thus affecting the energy barriers of interconversion between conformers, involving rotation around $\mathrm{CO}-\mathrm{OR}$ ).

The harmonic vibrational frequencies were calculated for all ETHPPE conformers, as well as for the corresponding diester E2THPPE (data available from the authors upon request). Table 2 comprises the calculated wavenumbers for the two most stable ETHPPE geometries, as well as for the lowest energy conformer of E2THPPE, showing a good overall agreement with both the experimental data and the theoretical values obtained by the authors for the analogous acid transTHPPE [24] and for trans-caffeic acid [37].

It is well known that this kind of phenolic compounds (either carboxylic acids or esters) occur predominantly as dimeric/oligomeric structures in the condensed phase, formed through intermolecular $(\mathrm{O}) \mathrm{H} \cdots \mathrm{O}(=\mathrm{C})$ interactions. Therefore, calculations were performed for dimeric species of transETHPPE and the analogous carboxylic acid trans-THPPE, in order to achieve a more accurate representation of these molecules in the solid state. The dimeric structure comprising two equal monomers of tETHPPE 1 (Fig. 4(a)) was found to display quite strong hydrogen close contacts between the carbonyl and the ring hydroxyl substituents- $(\mathrm{O}) \mathrm{H}_{22} \cdots$ $\mathrm{O}_{12}(=\mathrm{C})$ distances of $175 \mathrm{pm}$-yielding an overall geometry identical to the most stable dimer calculated for THPPE $((\mathrm{O}) \mathrm{H} \cdots \mathrm{O}(=\mathrm{C})$ equal to $180 \mathrm{pm}$, Fig. 4(b)). The presence of the ethyl ester groups in the tETHPPE 1 dimer is responsible for a tilted relative orientation of the two monomeric moieties as compared to the planar geometry of the THPPE dimer (Fig. 4(a) and (b)), in order to minimise steric repulsions. This type of close contacts between the ring hydroxyls and the carbonyl group are clearly favoured, even when top-to-top $\mathrm{H}$-bond interations between the terminal carboxylic functions may occur, in the phenolic acid analogues: for THPPE, for instance, the second lowest energy dimeric species was found to correspond to such an interaction (Fig. 4(c)) and displays a conformational energy $4.5 \mathrm{~kJ} \mathrm{~mol}^{-1}$ higher than the most stable dimer (with populations at room temperature equal to 16 and $84 \%$, respectively).

\subsection{Raman spectroscopy}

A complete assignment of the experimental bands of transETHPPE was carried out, based on its calculated vibrational frequencies (Table 2), as well as on experimental and theoretical results previously obtained for analogous compounds, such as caffeic acid [37] and THPPE [24]. The presence of the cis isomer is found to be negligible in the product synthesised as presently described, due to its much 


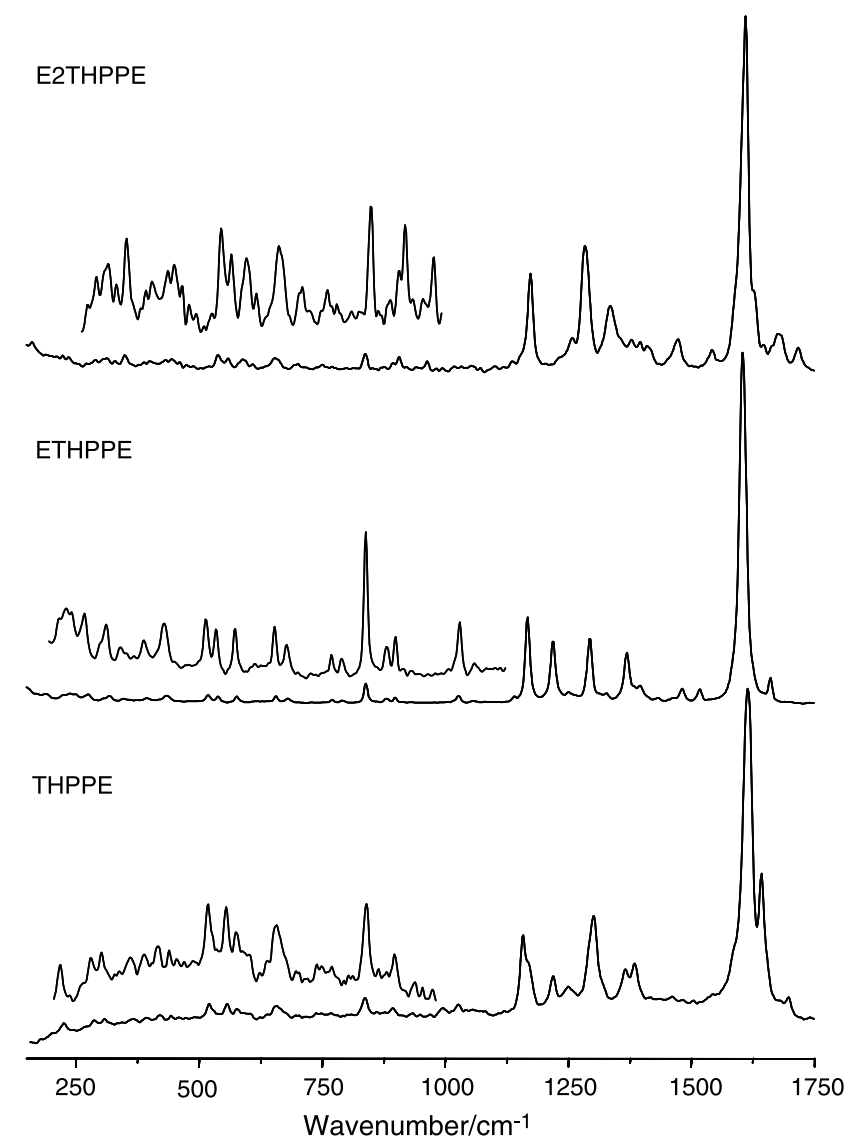

Fig. 5. Experimental Raman spectra $\left(100-1750 \mathrm{~cm}^{-1}\right)$ of trans-ethyl 3-(3,4,5tri-hydroxyphenyl)-2-propenoate (ETHPPE) and the corresponding acid (3-(3, 4,5-trihydroxyphenyl)-2-propenoic acid, THPPE) and diethyl ester (diethyl 2 (3,4,5-trihydroxyphenylmethylene)malonate, E2THPPE). (Solid state, $25^{\circ} \mathrm{C}$ ).

lower stability (which is corroborated by the theoretical results previously discussed).

Fig. 5 comprises the solid state experimental Raman spectra (from 100 to $1750 \mathrm{~cm}^{-1}$ ) of trans-ETHPPE, as well as of the corresponding acid trans-THPPE and the diester E2THPPE. The most characteristic features detected for solid ETHPPE were (Fig. 5, Table 2): (i) $\mathrm{OH}$ vibrations-stretching modes between ca. 3400 and $3300 \mathrm{~cm}^{-1}$, and deformations from 1360 to $1000 \mathrm{~cm}^{-1}$; (ii) $\mathrm{C}=\mathrm{O}$ stretching mode (with a rather low Raman scattering activity) -at $1660 \mathrm{~cm}^{-1}\left(1640 \mathrm{~cm}^{-1}\right.$ in the acid); (iii) $\mathrm{C}=\mathrm{C}$ stretching vibrations, both from the pendant carbon chain and the aromatic ring, yielding the most intense band in the spectrum (the latter displaying a higher Raman activity)—at ca. $1601 \mathrm{~cm}^{-1}$ (at $1612 \mathrm{~cm}^{-1}$ in the acid); (iv) $\mathrm{CH}$ vibrations-stretching modes, both symmetric and asymmetric, around 3000 and $2900 \mathrm{~cm}^{-1}$ respectively, and $\mathrm{CH}_{2} /$ $\mathrm{CH}_{3}$ deformations, between ca. 1475 and $1100 \mathrm{~cm}^{-1}$; (v) $\mathrm{CH}_{3}$ torsion, around $260 \mathrm{~cm}^{-1}$.

While for both the acid and the monoester the $\mathrm{C}=\mathrm{C}$ bonds from the carbon chain and the aromatic ring gave rise to an intense feature at 1612 and $1601 \mathrm{~cm}^{-1}$, respectively, two bands were observed for the diester, at 1608 and $1625 \mathrm{~cm}^{-1}$, assigned to $\nu_{\mathrm{C}=\mathrm{C} \text { (ring) }}$ and $\left(\nu_{\mathrm{C}=\mathrm{C} \text { (chain) }}+\nu_{\mathrm{C}=\mathrm{C} \text { (ring) }}\right)$ (Fig. 5, Table 2), the former having a much higher calculated Raman activity. Regarding the ester $\nu_{\mathrm{C}-\mathrm{O}}$ oscillator, a deviation to high wavenumbers was observed when going from THPPE (1132/ $1105 \mathrm{~cm}^{-1}$ calculated) to the esters ETHPPE $\left(1204 \mathrm{~cm}^{-1}\right.$ experimental vs $1248 \mathrm{~cm}^{-1}$ calculated) and E2THPPE (1271/ $1244 \mathrm{~cm}^{-1}$ experimental vs $1236 / 1222 / 1196 \mathrm{~cm}^{-1}$ calculated) (Table 2), which may be explained by the larger force constant of the $\mathrm{C}-\mathrm{O}$ bond in the latter, due to the enhancement of the inductive effect upon esterification. As to the $\nu_{\mathrm{C}=\mathrm{O}}$ vibrational mode, a shift to high frequencies was detected for the esters (Fig. 5): $1640 \mathrm{~cm}^{-1}$ for THPPE, $1660 \mathrm{~cm}^{-1}$ for ETHPPE, and 1717 and $1676 \mathrm{~cm}^{-1}$ for E2THPPE (respectively, in-phase and out-of-phase vibrations). This behaviour was not predicted by the calculations for the isolated molecule $-\nu_{\mathrm{C}=\mathrm{O}}$ at 1739,1719 and 1727/1712 for THPPE, ETHPPE and E2THPPE, respectively (Table 2)-since these do not consider the intermolecular close contacts occurring in the condensed phase and mostly affecting the modes involving the carbonyl and hydroxyl groups.

In fact, both the $\nu_{\mathrm{C}=\mathrm{O}}$ and $\nu_{\mathrm{OH}}$ oscillators were found to display a significant downward shift relative to the calculated values (Table 2), as expected according to the previous results on caffeic acid [37] and THPPE [24]. This is a consequence of the presence of dimers in the condensed phase, typical of this type of phenolic carboxylic acids/esters, where stabilising $(\mathrm{O}) \mathrm{H} \cdots \mathrm{O}(=\mathrm{C})$ intermolecular close contacts give rise to rather stable dimeric/oligomeric species [38,39] (Fig. 4). These findings are corroborated by the ab initio results obtained for both THPPE [24] and ETHPPE monomeric vs dimeric geometries: for the $\mathrm{OH}$ group involved in an intermolecular close contact in ETHPPE $\nu_{\mathrm{OH}}$ was shown to shift from ca. 3695 to $3430 \mathrm{~cm}^{-1}$ (in good agreement with the observed bands at $3399-3342 \mathrm{~cm}^{-1}$, Table 2). These $\nu_{\mathrm{OH}}$ vibrations usually yield broad Raman features (not easily detected experimentally), as a consequence of these intermolecular hydrogen close-contacts. The ester $\mathrm{C}=\mathrm{O}$ stretching $\left(\nu_{\mathrm{C}=\mathrm{O}}\right)$, also known to be affected by dimerisation, showed an expected deviation to low frequencies upon H-bond formation: from $1719 \mathrm{~cm}^{-1}$ in the monomer to $1682 / 1657 \mathrm{~cm}^{-1}$ in the dimeric species (in accordance with the experimental value of $1660 \mathrm{~cm}^{-1}$, Table 2). Furthermore, the bands ascribed to the $\mathrm{C}_{1}-\mathrm{O}_{14}$ stretching, and particularly to the inplane $\mathrm{O}_{14} \mathrm{H}_{22}$ bending mode, displayed a clear upward shift due to dimer formation: from 1366 and $1248 \mathrm{~cm}^{-1}$ (respectively) in the monomer to 1390 and $1440 \mathrm{~cm}^{-1}$ in the dimer. Consequently, the calculated wavenumbers associated to the groups prone to be involved in intermolecular hydrogen close-contacts (dimeric structures) presented a very good agreement with the experimental values obtained for the solid.

In view of better understanding the effect of dimerisation on the Raman pattern of ETHPPE, particularly on the $\nu_{\mathrm{C}=\mathrm{O}}$ oscillator, spectra were obtained for DMSO solutions. DMSO was chosen has a solvent since it is Raman-transparent in the spectral region of interest, and any possible interaction with the ester-via $(\mathrm{O}) \mathrm{H} \cdots \mathrm{O}(=\mathrm{S})$ close contacts, reflected in a band at ca. $1750 \mathrm{~cm}^{-1}$ [40]—is known to occur only for very high 

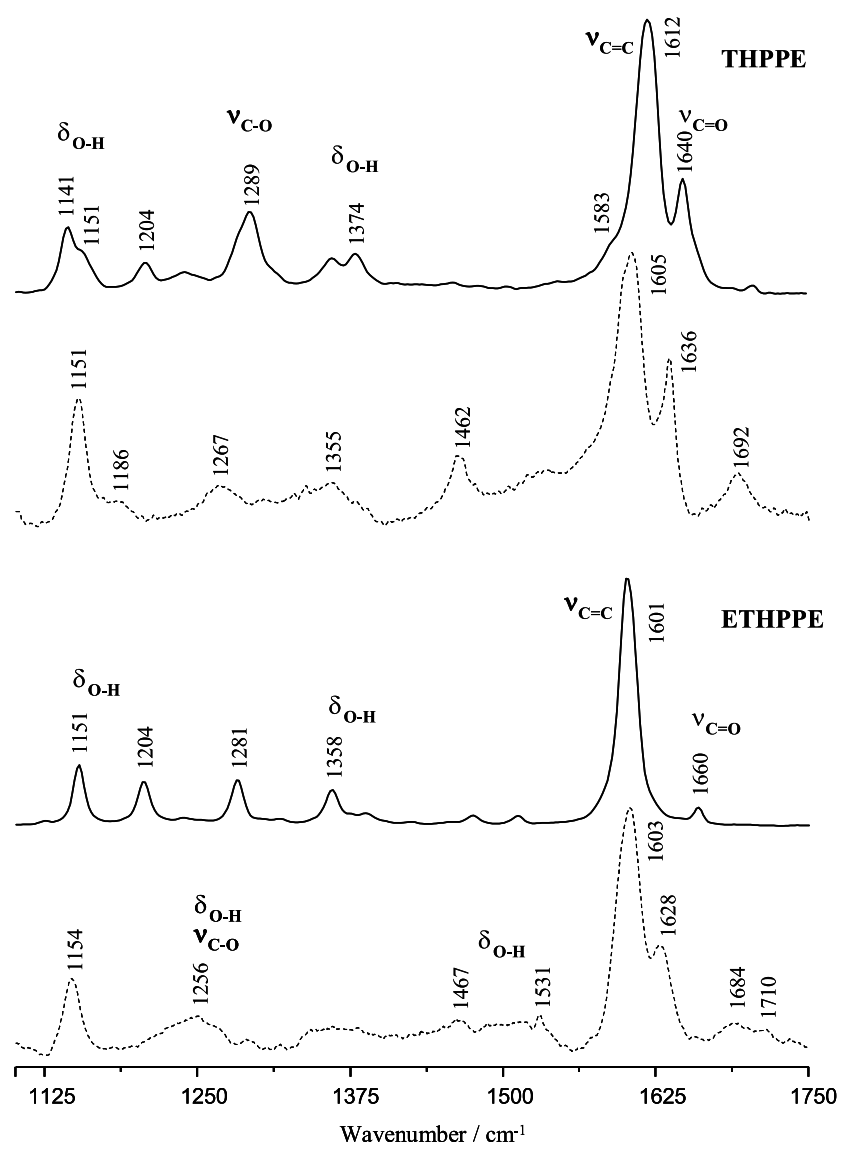

Fig. 6. Experimental Raman spectra $\left(1100-1750 \mathrm{~cm}^{-1}\right.$, at $\left.25^{\circ} \mathrm{C}\right)$ of trans-ethyl 3-(3,4,5-trihydroxyphenyl)-2-propenoate (ETHPPE) and the corresponding acid trans-3-(3,4,5-trihydroxyphenyl)-2-propenoic acid (THPPE), both in the solid state and in DMSO $40 \mathrm{mM}$ solution. (Solid line-solid state; dotted linesolution).

solvent:solute ratios (much higher than the ones used along this study).

While for the solid the $\mathrm{C}=\mathrm{O}$ group gave rise to only one stretching band, at $1660 \mathrm{~cm}^{-1}$, for ETHPPE-DMSO $40 \mathrm{mM}$ solution two features were assigned to $\nu_{\mathrm{C}=\mathrm{O}}$, at 1710 and $1684 \mathrm{~cm}^{-1}$ (Fig. 6), the former being slightly enhanced in the $20 \mathrm{mM}$-solution. Considering that the dimer:monomer ratio decreases upon dilution, i.e. when going from the solid sample to the solution, these two bands were ascribed to the free and hydrogen-bonded forms of the $\mathrm{C}=\mathrm{O}$ group, respectively (in analogy with the THPPE system previously studied [24]). In fact, the higher relative population of monomeric species in the solution is reflected in the observation of free carbonyl groups, not involved in intermolecular $\mathrm{H}$-bonds $-\nu_{\mathrm{C}=\mathrm{O}}$ mode at $1710 \mathrm{~cm}^{-1}$ in agreement with the value of $1719 \mathrm{~cm}^{-1}$ calculated for the isolated molecule (Table 2). The H-bonded $\mathrm{C}=\mathrm{O}$ groups give rise to a stretching band at $1684 \mathrm{~cm}^{-1}$, which corresponds to the band at $1660 \mathrm{~cm}^{-1}$ detected for the solid. The intensity increase of the $1710 \mathrm{~cm}^{-1}$ feature upon dilution corroborates this hypothesis. When compared to the substituted benzaldehydes for which this type of dimeric structures has been initially reported [41,42], the frequency shift observed for ETHPPE (and analogous systems) is quite larger, on account of the higher electronic delocalisation occuring in these hydroxycinnamic derivatives due to the presence of the unsaturated linear carbon chain: ca. $10 \mathrm{~cm}^{-1} \mathrm{vs}$ ca. $50 \mathrm{~cm}^{-1}$, respectively.

The $\mathrm{C}-\mathrm{O}$ stretching vibration also showed to be affected by this dimer-monomer equilibrium, displaying a shift from $1281 \mathrm{~cm}^{-1}$ in the solid, to $1256 \mathrm{~cm}^{-1}$ in the solution (Fig. 6). The typical phenolic $\mathrm{OH}$ bending vibration at about $1358 \mathrm{~cm}^{-1}$ was also found to suffer changes upon dimerisation (from the solution to the solid state, Fig. 6), which is easily understandable in view of the involvement of some of these hydroxyl groups in the dimerisation process (Fig. 4). The $\nu_{\mathrm{C}=\mathrm{C}}$ mode from the carbon chain, in turn, undergoes an upward shift when going from the dimer $\left(1601 \mathrm{~cm}^{-1}\right)$ to the monomer $\left(1628 \mathrm{~cm}^{-1}\right)$, while the ring $\nu_{\mathrm{C}=\mathrm{C}}$ appears at roughly the same frequency $\left(1603 \mathrm{~cm}^{-1}\right)$. Moreover, a new feature is detected in the spectrum of the solution at $1256 \mathrm{~cm}^{-1}$ (Fig. 6), which is assigned to both $\mathrm{C}_{1}-\mathrm{O}_{14}$ stretching and in-plane $\mathrm{C}_{1}-\mathrm{O}_{14}-\mathrm{H}_{22}$ deformation-thus being directly affected by the formation of dimeric structures such as the one represented in Fig. 4.

\section{Conclusion}

The conformational analysis performed for ETHPPE rendered eighteen distinct conformers, twelve for the trans isomer and six for the cis species, with structural differences concerning the conformation of the ethyl ester group and the adjacent unsaturated carbon chain, as well as the orientation of the three ring hydroxyl substituents. Two of these conformers-tETHPPE $1(\Delta E=0)$ and tETHPPE $2(\Delta E=$ $0.6 \mathrm{~kJ} \mathrm{~mol}^{-1}$ )—were found to be particularly stable, with populations at room temperature of 47 and $37 \%$, respectively. The conformational preferences of this molecule, mainly determined by an effective $\pi$-electron delocalisation coupled to a minimisation of repulsive interactions, were found to be: a planar geometry; an S-cis orientation of the ester group; a $\left(\mathrm{C}_{11} \mathrm{C}_{10} \mathrm{C}_{9} \mathrm{C}_{3}\right)$ dihedral equal to $180^{\circ}$; an identical orientation of the hydroxyl groups, coplanar with the ring; a syn conformation of the carbonyl group and the ring OH's. The preference for planarity was expected, since it favours electron delocalisation through the expanded $\pi$ system of the hyperconjugated molecule of ETHPPE. Thus, non-planar geometries arose only in order to overcome steric hindrance destabilising factors (such as $\mathrm{H} \cdots \mathrm{H}$ interactions), whose minimisation resulted to be more favourable then the maintenance of planarity.

Comparison of the conformational results presently described with the ones reported for THPPE allows to conclude that the additional degrees of freedom introduced by esterification are mainly reflected in the internal rotation around the $\mathrm{O}_{13}-\mathrm{C}_{11}$ bond, which showed to be the most important factor determining the overall stability of the ETHPPE molecule. Also, the S-cis to S-trans energy difference was found to be almost unaffected by the presence of the ethyl ester group as compared to the carboxylic function $(\Delta E$ ca. $11.5 \mathrm{~kJ} \mathrm{~mol}^{-1}$ for both ETHPPE and THPPE). Furthermore, the conformational analysis performed for the analogous dihydroxylated cinnamic acid (CA) [37] evidences that 
inclusion of a third $\mathrm{OH}$ ring substituent (as in THPPE and ETHPPE) does not significantly affect the structural preferences described for this kind of phenolic systems.

A complete assignment of the solid state Raman spectrum of trans-ETHPPE was carried out, in the light of the ab initio calculations and the reported data on the analogous compounds THPPE [24] and caffeic acid [37]. A quite good agreement was found between the results now obtained for ETHPPE-both calculated and experimental vibrational wavenumbers-and the data found in the literature for similar molecules, both from theoretical and spectroscopic studies [24,37-39,43,44].

In conclusion, this kind of conformational analysis carried out for phenolic derivatives, through ab initio theoretical calculations coupled to vibrational spectroscopy, are of the utmost importance for understanding the structure-activity relationships (SAR's) ruling the biological activity of such compounds. In fact, the hydroxycinnamic acid derivatives presently studied were found to display both antioxidant [21] and cytotoxic properties against human cancer cell lines $[22,23]$, that can only be accurately interpreted in the light of the corresponding conformational analysis.

\section{Acknowledgements}

The authors thank the Chemistry Department of the University of Aveiro, where the FT-Raman experiments were carried out. RC acknowledges financial support from FCT$\mathrm{PhD}$ fellowship SFRH/BD/16520/2004.

\section{References}

[1] O.I. Aruoma, A. Murcia, J. Butler, B. Halliwell, J. Agric. Food Chem. 41 (1993) 1880

[2] T. Nakayama, Cancer Res. 54 (1994) 1991 Suppl..

[3] C.A. Rice-Evans, N.J. Miller, G. Paganga, Free Radic. Biol. Med. 20 (1996) 933.

[4] G. Cao, E. Sofic, L. Prior, Free Radic. Biol. Med. 22 (1997) 749.

[5] B. Halliwell, J.C. Gutteridge, Free Radicals in Biology and Medicine, Oxford Science Publications, 1999.

[6] E. Sergediene, K. Jonsson, H. Szymusiak, B. Tyrakowska, I.M.C.M. Rietjens, N. Cenas, FEBS Lett. 462 (1999) 392.

[7] M. Inoue, N. Sakaguchi, K. Isuzugawa, H. Tani, Y. Ogihara, Biol. Pharm. Bull. 23 (2000) 1153 (and refs. therein).

[8] G. Roy, M. Lombardía, C. Palacios, A. Serrano, C. Cespón, E. Ortega, P. Eiras, S. Lujan, Y. Revilla, P. González-Porqué, Arch. Biochem. Biophys. 383 (2000) 206 (and refs. therein).

[9] T. Gao, Y. Ci, H. Jian, C. An, Vibr. Spectrosc. 24 (2000) 225.

[10] W.Y. Chung, Y.J. Jung, Y.J. Surh, S.S. Lee, K. Park, Mutat. Res. 496 (2001) 199.

[11] A. Russo, R. Longo, A. Vanella, FitoterapiaSuppl. 1(2002) S21.

[12] C. Anselmi, M. Centini, P. Granata, A. Sega, A. Buonocore, A. Bernini, R.M. Facino, J. Agric. Food. Chem. 52 (2004) 6425.

[13] Y.T. Lee, M.J. Don, C.H. Liao, H.W. Chiou, C.F. Chen, L.K. Ho, Clin. Chim. Acta. 352 (2005) 135.

[14] G.J. Kelloff, Adv. Cancer Res. 78 (2000) 199.

[15] N.N. Mahmoud, A.M. Carothers, D. Grunberger, R.T. Bilinski, M.R. Churchill, C. Martucci, H.L. Newmark, M.M. Bertagnolli, Adv. Cancer Res. 21 (2000) 921.
[16] A. Serrano, C. Palacios, G. Roy, C. Cespón, M.L. Villar, M. Nocito, P. González-Porqué, Arch. Biochem. Biophys. 350 (1) (1998) 49.

[17] F. Natella, M. Nardini, M. Di Felice, C. Scaccini, J. Agric. Food Chem. 47 (1999) 1453.

[18] F.A.M. Silva, F. Borges, C. Guimarães, J.L.F.C. Lima, C. Matos, S. Reis, J. Agric. Food Chem. 48 (2000) 2122.

[19] A. Hosoda, Y. Ozaki, A. Kashiwada, M. Mutoh, K. Wakabayashi, E. Nomura, H. Taniguchi, Bioorg. Med. Chem. 10 (2002) 1189.

[20] A.Y.T. Lee, M.J. Don, P.S. Hung, Y.C. Shen, Y.S. Lo, K.W. Chang, C.F. Chen, L.K. Ho, Cancer Lett. 223 (2005) 19.

[21] V. Rio, C. Siquet, S. Reis, M.P.M. Marques, J.B. Sousa, F. Borges, Cancer Lett. submitted.

[22] C.A. Gomes, T. Girão da Cruz, J.L. Andrade, N. Milhazes, F. Borges, M.P.M. Marques, J. Med. Chem. 46 (2003) 5395.

[23] S.M. Fiuza, C. Gomes, L.J. Teixeira, M.T. Girão da Cruz, M.N.D.S. Cordeiro, N. Milhazes, F. Borges, M.P.M. Marques, Bioorg. Med. Chem 12 (2004) 3581.

[24] S.M. Fiuza, E. Van Besien, N. Milhazes, F. Borges, M.P.M. Marques, J. Mol. Struct. 693 (2004) 103.

[25] K. Freudenberg, H.H. Hubner, Chem. Ber. 85 (1952) 1181.

[26] M.J. Frisch, G.W. Trucks, H.B. Schlegel, G.E. Scuseria, M.A. Robb, J.R. Cheeseman, V.G. Zakrzewski, J.A. Montgomery Jr., R.E. Stratmann, J.C. Burant, S. Dapprich, J.M. Millam, A.D. Daniels, K.N. Kudin, M.C. Strain, O. Farkas, J. Tomasi, V. Barone, M. Cossi, R. Cammi, B. Mennucci, C. Pomelli, C. Adamo, S. Clifford, J. Ochterski, G.A. Petersson, P.Y. Ayala, Q. Cui, K. Morokuma, D.K. Malick, A.D. Rabuck, K. Raghavachari, J.B. Foresman, J. Cioslowski, J.V. Ortiz, A.G. Baboul, B.B. Stefanov, G. Liu, A. Liashenko, P. Piskorz, I. Komaromi, R. Gomperts, R.L. Martin, D.J. Fox, T. Keith, M.A. AlLaham, C.Y. Peng, A. Nanayakkara, M. Challacombe, P.M.W. Gill, B. Johnson, W. Chen, M.W. Wong, J.L. Andres, C. Gonzalez, M. HeadGordon, E.S. Replogle, J.A. Pople, Gaussian 98, Revision A.9, Gaussian Inc., Pittsburgh PA, USA, 1998.

[27] C. Lee, W. Yang, R.G. Parr, Phys. Rev. B 37 (1988) 785.

[28] B. Miehlich, A. Savin, H. Stoll, H. Preuss, Chem. Phys. Lett. 157 (1989) 200.

[29] A. Becke, Phys. Rev. A 38 (1988) 3098.

[30] A. Becke, J. Chem. Phys. 98 (1993) 5648.

[31] G.A. Petersson, A. Bennett, T.G. Tensfeldt, M.A. Al-Laham, W.A. Shirley, J. Mantzaris, J. Chem. Phys. 89 (1988) 2193.

[32] A.P. Scott, L. Radom, J. Phys. Chem. 100 (1996) 16502.

[33] S.F. Boys, F. Bernardi, Molec. Phys. 19 (1970) 553.

[34] C. Peng, P.Y. Ayala, H.B. Schlegel, J. Frisch, Comput. Chem. 17 (1996) 49.

[35] S. García-Granda, G. Beurskens, P.T. Beurskens, T.S.R. Krishna, G.R. Desiraju, Acta Cryst. C43 (1987) 683.

[36] E. Bakalbassis, A. Chatzopoulou, V.S. Melissas, M. Tsimidou, M. Tsolaki, A. Vafiadis, Lipids 36 (2001) 181.

[37] E. van Besien, M.P.M. Marques, J. Mol. Struct.(THEOCHEM) 625 (2003) 265.

[38] S. Sánchez-Cortés, V. García-Ramos, Spectrochim. Acta A55 (1999) 2935.

[39] S. Sánchez-Cortés, J.V. García-Ramos, Appl. Spec. 54 (2000) 230.

[40] P. Novak, D. Vikic-Topic, Z. Meic, S. Sekusak, A. Sabljic, J. Mol. Struct. 356 (1995) 131.

[41] N. Karger, A.M. Amorim da Costa, P.J.A. Ribeiro-Claro, J. Phys. Chem. A 103 (1999) 8672

[42] M.P.M. Marques, A.M. Amorim da Costa, P.J.A. Ribeiro-Claro, J. Phys. Chem. A 105 (2001) 5292.

[43] S. Sánchez-Cortés, J.V. García-Ramos, J. Colloid Interface Sci. 231 (2000) 98 .

[44] R. Calheiros, N. Milhazes, F. Borges, M.P.M. Marques, J. Mol. Struct. 692 (2004) 91. 